\title{
Systems of Semilinear Parabolic Variational Inequalities with Time-Dependent Convex Obstacles
}

\author{
Tomasz Klimsiak ${ }^{1,2}$ - Andrzej Rozkosz ${ }^{2}$. \\ Leszek Słomiński
}

(C) The Author(s) 2016. This article is published with open access at Springerlink.com

\begin{abstract}
We consider a system of seminlinear parabolic variational inequalities with time-dependent convex obstacles. We prove the existence and uniqueness of its solution. We also provide a stochastic representation of the solution and show that it can be approximated by the penalization method. Our proofs are based upon probabilistic methods from the theory of Markov processes and the theory of backward stochastic differential equations.
\end{abstract}

Keywords Semilinear variational inequality · Divergence form operator · Time-dependent obstacle $\cdot$ Penalization method - Reflected backward stochastic differential equation

Mathematics Subject Classification Primary 35K87 · Secondary 60H30

\section{Introduction}

Let $E$ be a bounded domain in $\mathbb{R}^{d}, E_{T}=[0, T] \times E$ and let $D=\left\{D(t, x),(t, x) \in E_{T}\right\}$ be a family of uniformly bounded closed convex sets in $\mathbb{R}^{m}$ with nonempty interiors. In

Andrzej Rozkosz

rozkosz@mat.umk.pl

Tomasz Klimsiak

tomas@mat.umk.pl

Leszek Słomiński

leszeks@mat.umk.pl

1 Institute of Mathematics, Polish Academy of Sciences, Śniadeckich 8, 00-956 Warsaw, Poland

2 Faculty of Mathematics and Computer Science, Nicolaus Copernicus University, Chopina 12/18, 87-100 Toruń, Poland 
the paper we study the problem, which roughly speaking can be stated as follows: for measurable function $\varphi: E \rightarrow \mathbb{R}^{m}$ such that $\varphi(x) \in D(T, x), x \in E$, and measurable $f: E_{T} \times \mathbb{R}^{m} \times \mathbb{R}^{m \times d} \rightarrow \mathbb{R}^{m}$ find $u=\left(u^{1}, \ldots, u^{m}\right): E_{T} \rightarrow \mathbb{R}^{m}$ such that

$$
u(t, x) \in D(t, x) \text { for }(t, x) \in E_{T}, \quad u(T, \cdot)=\varphi, \quad u(t, \cdot)_{\mid \partial E}=0, t \in(0, T)
$$

and

$$
\int_{0}^{T}\left(\frac{\partial u}{\partial t}+L_{t} u+f_{u}, v-u\right)_{H} d t \geq 0
$$

for every $v=\left(v^{1}, \ldots, v^{m}\right)$ such that $v^{i} \in L^{2}\left(0, T ; H_{0}^{1}(E)\right), i=1, \ldots, m$, and $v(t, x) \in D(t, x)$ for $(t, x) \in E_{T}$. In $(1.2),(\cdot, \cdot)_{H}$ is the usual inner product in $H=\left[L^{2}(E)\right]^{m}$,

$$
f_{u}(t, x)=f\left(t, x, u(t, x), \sigma \nabla u^{1}(t, x), \ldots, \sigma \nabla u^{m}(t, x)\right), \quad(t, x) \in E_{T}
$$

and

$$
L_{t}=\frac{1}{2} \sum_{i, j=1}^{d} \frac{\partial}{\partial x_{j}}\left(a_{i j}(t, x) \frac{\partial}{\partial x_{i}}\right)
$$

where $a: E_{T} \rightarrow \mathbb{R}^{d} \otimes \mathbb{R}^{d}$ is a measurable symmetric matrix-valued function such that for some $\Lambda \geq 1$,

$$
\Lambda^{-1}|y|^{2} \leq \sum_{i, j=1}^{d} a_{i j}(t, x) y_{i} y_{j} \leq \Lambda|y|^{2}, \quad y \in \mathbb{R}^{d}
$$

for a.e. $(t, x) \in E_{T}$. By putting $a(t, x)=a(0, x)$ for $t \notin[0, T], x \in E$ we can and will assume that $a$ is defined and satisfies (1.4) in all $\mathbb{R} \times E$. In (1.3), $\sigma$ is the symmetric square root of $a$.

The main feature of the paper is that we deal with time-dependent obstacles. In case of single equation, i.e. when $m=1$, problem (1.1), (1.2) is quite well investigated. For various results on existence, uniqueness and approximation of solutions in case of $L^{2}$ data and one or two regular obstacles, i.e. when $D$ has the form $D(t, x)=\{y \in$ $\mathbb{R}: \underline{h}(t, x) \leq y \leq \bar{h}(t, x)\}$ for some regular $\underline{h}, \bar{h}: E_{T} \rightarrow \overline{\mathbb{R}}$ (possibly $\underline{h} \equiv-\infty$ or $\bar{h} \equiv+\infty)$ see the monograph [2, Sects. 2.2, 2.18] and more recent papers $[4,13,15]$. Linear problem of the form (1.1), (1.2) with $L^{2}$ data and one irregular barrier is investigated in [20,26]. For recent results on semilinear problem see [8] (one merely measurable obstacle) and [9] (two measurable obstacles satisfying some separation condition). The problem with two irregular obstacles and $L^{1}$ data is investigated in [11]. 
In case of systems of equations the situation is quite different. To our knowledge, in this case only few partial results exist (see [20, Sect. 1.2] for the existence of solutions of weakly coupled systems and Example 9.3 and Theorem 9.2 in [18, Chap. 2] for the special case $0 \in D\left(t_{2}, \cdot\right) \subset D\left(t_{1}, \cdot\right)$ if $0 \leq t_{1} \leq t_{2}$; see also [14] for existence results concerning a different but related problem). The aim of the present paper is to prove quite general results on existence, uniqueness and approximation of solutions of (1.1), (1.2) in case the data are square integrable and $D$ satisfies some mild regularity assumptions. The case of $L^{1}$ data and irregular obstacles is more difficult but certainly deserves further investigation.

In our opinion one of the main problem one encounters when dealing with systems and time-dependent obstacles lies in the proper choice of the definition of a solution. In fact, the main problem is to adopt definition which ensures uniqueness of solutions. The definition used in the present paper is a natural extension to systems of the definition used in one-dimensional case in [26] and then in [8,9,11]. By a solution of (1.1), (1.2) we mean a pair $(u, \mu)$ consisting of a function $u=\left(u^{1}, \ldots, u^{m}\right): E_{T} \rightarrow \mathbb{R}^{m}$ and vector $\mu=\left(\mu^{1}, \ldots \mu^{m}\right)$ of signed Borel measures on $E_{T}$ satisfying the following three conditions:

(a) $u^{i}$ are quasi-continuous (with respect to the parabolic capacity determined by $\left.L_{t}\right)$ functions of class $C([0, T] ; H) \cap L^{2}\left(0, T ; H_{0}^{1}(E)\right)$ and $\mu^{i}$ are smooth (with respect to the same capacity) measures of finite variation,

(b) $u$ is a weak solution of the problem

$$
\frac{\partial u}{\partial t}+L_{t} u=-f_{u}-\mu, \quad u(T, \cdot)=\varphi, \quad u(t, \cdot)_{\mid \partial E}=0, t \in(0, T),
$$

such that $u(t, x) \in D(t, x)$ for quasi-every (q.e. for short) $(t, x) \in(0, T] \times E$, (c) for every quasi-continuous function $h=\left(h^{1}, \ldots, h^{m}\right)$ such that $h(t, x) \in D(t, x)$ for q.e $(t, x) \in(0, T] \times E$,

$$
\sum_{i=1}^{m} \int_{t}^{T} \int_{E}\left(u^{i}-h^{i}\right) d \mu^{i} \leq 0, \quad t \in(0, T)
$$

In the above definition $\mu$ may be called the "obstacle reaction measure". It may be interpreted as the energy we have to add to the system to keep the solution inside $D$. Condition (c) is some kind of minimality condition imposed on $\mu$. In case $m=1$ it reduces to the usual minimality condition saying that $\mu=\mu^{+}-\mu^{-}$, where $\mu^{+}$ (resp. $\mu^{-}$) is a positive measure acting only when $u$ is equal to the lower obstacle $\underline{h}$ (resp. upper obstacle $\bar{h}$ ). Also remark that an important requirement in our definition is that $u$ is quasi-continuous and $\mu$ is smooth. It not only ensures that the integral in (c) is meaningful, but also allows us to give a probabilistic representation of solutions. In fact, this probabilistic representation may serve as an equivalent definition of a solution of (1.1), (1.2).

As in classical monographs $[2,5,17,18]$, and papers $[14,15,20]$, in the present paper we work in $L^{2}$ setting. We assume that $\varphi \in\left[L^{2}(E)\right]^{m}, f(\cdot, \cdot, 0,0) \in$ $L^{2}\left(0, T ;\left[L^{2}(E)\right]^{m}\right)$ and $f(t, x, \cdot, \cdot)$ is Lipschitz continuous for $(t, x) \in E_{T}$. An 
important, model example of operator $L_{t}$ satisfying (1.4) is the Laplace operator. But as in $[17,18,20]$, to cover classic examples, like temperature control in domains with discontinuous coefficient of thermal conductivity (see [17, Chap. 1, §3.4], [5, Chap. I, §3.3, 4.4]), in the paper we consider divergence form operator with possibly discontinuous $a$. As for $D$, we assume that $(t, x) \mapsto D(t, x) \in$ Conv is continuous if we equip Conv with the Hausdorff metric. We also assume that $D$ satisfies the following separation condition: one can find a solution $u_{*} \in \mathcal{W}$ of the Cauchy problem

$$
\frac{\partial u_{*}}{\partial t}+L_{t} u_{*}=-f_{*}, \quad u_{*}(T)=\varphi_{*}
$$

with some $\varphi_{*} \in\left[L^{2}(E)\right]^{m}, f_{*} \in L^{2}\left(0, T ;\left[L^{2}(E)\right]^{m}\right)$ such that $u_{*}(t, x) \in D^{*}(t, x)$ for q.e. $(t, x) \in E_{T}$, where $D^{*}(t, x)=\{y \in D(t, x): \operatorname{dist}(y, \partial D(t, x)) \geq \varepsilon\}$ for some $\varepsilon>0$. We show that under the above assumptions there exists a unique solution $(u, \mu)$ of $(1.1),(1.2)$ and that $u$ and $\mu$ may be approximated by the penalization method. Note that our separation condition is not optimal, because we assume that $\varepsilon>0$ and that $u_{*}$ is more regular than the solution $u$ itself [see condition (a)]. The condition is also stronger than known sufficient separation conditions in the one dimensional case (see [9]). Nevertheless, it is satisfied in many interesting situations.

As in $[8,9,11]$, to prove our result we use probabilistic methods. In particular, we rely heavily on the results of our earlier paper [12] devoted to reflected backward stochastic differential equations with time-dependent obstacles and in proofs we use the methods of the theory of Markov processes and probabilistic potential theory. Also note that the first results on multidimensional reflected backward stochastic differential equations were proved in [7] in case $D$ is a fixed convex domain. The results of [7] were generalized in [25] to equations with Wiener-Poisson filtration. For related results with some time-depending domains see the recent paper [22].

\section{Preliminaries}

For $x \in \mathbb{R}^{m}, z \in \mathbb{R}^{m \times d}$ we set $|x|^{2}=\sum_{i=1}^{m}\left|x_{i}\right|^{2},\|z\|^{2}=\operatorname{trace}\left(z^{*} z\right)$. By $\langle\cdot, \cdot\rangle$ we denote the usual scalar product in $\mathbb{R}^{m}$. Given a Hilbert space $\mathcal{H}$ we denote by $[\mathcal{H}]^{m}$ its product equipped with the usual inner product $(u, v)_{[\mathcal{H}]^{m}}=\sum_{i=1}^{m}\left(u^{i}, v^{i}\right)_{\mathcal{H}}$ and norm $\|u\|_{[\mathcal{H}]^{m}}=\left((u, u)_{[\mathcal{H}]^{m}}\right)^{1 / 2}$. We will identify the space $\left[L^{2}(0, T ; \mathcal{H})\right]^{m}$ with $L^{2}\left(0, T ;[\mathcal{H}]^{m}\right)$.

Throughout the paper, $E$ is a nonempty bounded connected open subset of $\mathbb{R}^{d} \cdot \bar{E}$ is the closure of $E$ in $\mathbb{R}^{d}, E^{1}=\mathbb{R} \times E, E_{T}=[0, T] \times E, E_{0, T}=(0, T] \times E$. We set $H=\left[L^{2}(E)\right]^{m}, V=\left[H_{0}^{1}(E)\right]^{m}, V^{\prime}=\left[H^{-1}(E)\right]^{m}$, where $H^{-1}(E)$ is the dual of the Sobolev space $H_{0}^{1}(E)$. For $u \in V$ we set $\|\nabla u\|_{H}^{2}=\sum_{k=1}^{d} \sum_{i=1}^{m} \|$ $\frac{\partial u^{i}}{\partial x_{k}} \|_{L^{2}(E)}^{2}$.

The Lebesgue measure on $\mathbb{R}^{d}$ will be denoted by $m$. By $m_{1}$ we denote the Lebesgue measure on $E^{1}$. 


\subsection{Convex Sets and Functions}

By Conv we denote the space of all bounded closed convex subsets of $\mathbb{R}^{m}$ with nonempty interiors endowed with the Hausdorff metric $\rho$, that is for any $D, G \in$ Conv we set

$$
\rho(D, G)=\max \left(\sup _{x \in D} \operatorname{dist}(x, G), \sup _{x \in G} \operatorname{dist}(x, D)\right),
$$

where $\operatorname{dist}(x, D)=\inf _{y \in D}|x-y|$.

Let $D \in$ Conv and let $\mathcal{N}_{y}$ denote the set of inward normal unit vectors at $y \in \partial D$. It is well known (see, e.g., [19]) that $\mathbf{n} \in \mathcal{N}_{y}$ if and only if $\langle y-x, \mathbf{n}\rangle \leq 0$ for every $x \in D$. If moreover $a \in \operatorname{Int} D$ then for every $\mathbf{n} \in \mathcal{N}_{y}$,

$$
\langle y-a, \mathbf{n}\rangle \leq-\operatorname{dist}(a, \partial D) .
$$

If $\operatorname{dist}(x, D)>0$ then there exists a unique $y=\Pi_{D}(x) \in \partial D$ such that $|y-x|=$ $\operatorname{dist}(x, D)$. One can observe that $(y-x) /|y-x| \in \mathcal{N}_{y}$. Moreover (see [19]), for every $a \in \operatorname{Int} D$,

$$
\langle x-a, y-x\rangle \leq-\operatorname{dist}(a, \partial D)|y-x| .
$$

Also note that for any nonempty bounded closed convex sets $D, G \subset \mathbb{R}^{m}$ and any $x, y \in \mathbb{R}^{m}$,

$$
\left|\Pi_{D}(x)-\Pi_{G}(y)\right|^{2} \leq|x-y|^{2}+2[\operatorname{dist}(x, D)+\operatorname{dist}(y, G)] \rho(D, G)
$$

(see [21, Chap. 0, Proposition 4.7]).

\subsection{Time-Dependent Dirichlet Forms}

Let $\mathcal{A}=\{a(t ; \cdot, \cdot), t \in \mathbb{R}\}$ be the family of bilinear forms on $H_{0}^{1}(E) \times H_{0}^{1}(E)$ defined as

$$
a(t ; \varphi, \psi)=\frac{1}{2} \sum_{i, j=1}^{d} \int_{E} a_{i j}(t, x) \frac{\partial \varphi}{\partial x_{i}} \frac{\partial \psi}{\partial x_{j}} d x
$$

and let $\mathcal{V}=L^{2}\left(\mathbb{R} ; H_{0}^{1}(E)\right), \mathcal{V}^{\prime}=L^{2}\left(\mathbb{R} ; H^{-1}(E)\right), \mathcal{W}=\left\{u \in \mathcal{V}: \frac{\partial u}{\partial t} \in \mathcal{V}^{\prime}\right\}$ We equip $\mathcal{W}$ with the usual norm $\|u\|_{\mathcal{W}}=\|u\|_{\mathcal{V}}+\left\|\frac{\partial u}{\partial t}\right\|_{\mathcal{V}^{\prime}}$. By $\mathcal{E}$ we denote the time-dependent form determined by $\mathcal{A}$, i.e.

$$
\mathcal{E}(u, v)= \begin{cases}\left\langle-\frac{\partial u}{\partial t}, v\right\rangle+\int_{\mathbb{R}} a(t ; u(t), v(t)), & u \in \mathcal{W}, v \in \mathcal{V}, \\ \left\langle\frac{\partial v}{\partial t}, u\right\rangle+\int_{\mathbb{R}} a(t ; u(t), v(t)), & u \in \mathcal{V}, v \in \mathcal{W}\end{cases}
$$

where $\langle\cdot, \cdot\rangle$ is the duality pairing between $\mathcal{V}^{\prime}$ and $\mathcal{V}$. 
For $T>0$ we set $\mathcal{H}_{0, T}=L^{2}\left(0, T ; L^{2}(E ; m)\right), \mathcal{V}_{0, T}=L^{2}\left(0, T ; H_{0}^{1}(E)\right), \mathcal{V}_{0, T}^{\prime}=$ $L^{2}\left(0, T ; H^{-1}(E)\right), \mathcal{W}_{0, T}=\left\{u \in \mathcal{V}_{0, T}: \frac{\partial u}{\partial t} \in \mathcal{V}_{0, T}^{\prime}\right\}$ and $\mathcal{W}_{0}=\left\{u \in \mathcal{W}_{0, T}: u(0)=\right.$ $0\}, \mathcal{W}_{T}=\left\{u \in \mathcal{W}_{0, T}: u(T)=0\right\}$. By $\mathcal{E}^{0, T}$ we denote the time-dependent form defined as

$$
\mathcal{E}^{0, T}(u, v)= \begin{cases}\left\langle-\frac{\partial u}{\partial t}, v\right\rangle+\int_{0}^{T} a(t ; u(t), v(t)), & u \in \mathcal{W}_{T}, v \in \mathcal{V}_{0, T} \\ \left\langle\frac{\partial v}{\partial t}, u\right\rangle+\int_{0}^{T} a(t ; u(t), v(t)), & u \in \mathcal{V}_{0, T}, v \in \mathcal{W}_{0}\end{cases}
$$

where now $\langle\cdot, \cdot\rangle$ denote the duality pairing between $\mathcal{V}_{0, T}^{\prime}$ and $\mathcal{V}_{0, T}$. Note that the forms $\mathcal{E}, \mathcal{E}^{0, T}$ can be identified with some generalized Dirichlet form (see [28, Example I.4.9.(iii)]).

By Propositions I.3.4 and I.3.6 in [28] the form $\mathcal{E}^{0, T}$ determines uniquely a strongly continuous resolvents $\left(G_{\alpha}^{0, T}\right)_{\alpha>0},\left(\hat{G}_{\alpha}^{0, T}\right)_{\alpha>0}$ on $\mathcal{H}_{0, T}$ such that $\left(G_{\alpha}^{0, T}\right)_{\alpha>0},\left(\hat{G}_{\alpha}^{0, T}\right)_{\alpha>0}$ are sub-Markov, $G_{\alpha}^{0, T}\left(\mathcal{H}_{0, T}\right) \subset \mathcal{W}_{T}, \hat{G}_{\alpha}^{0, T}\left(\mathcal{H}_{0, T}\right) \subset \mathcal{W}_{0}$, and

$$
\mathcal{E}_{\alpha}^{0, T}\left(G_{\alpha}^{0, T} \eta, u\right)=(u, \eta)_{\mathcal{H}_{0, T}}, \quad \mathcal{E}_{\alpha}^{0, T}\left(u, \hat{G}_{\alpha}^{0, T} \eta\right)=(u, \eta)_{\mathcal{H}_{0, T}}
$$

for $u \in \mathcal{V}_{0, T}, \eta \in \mathcal{H}_{0, T}$, where $\mathcal{E}_{\alpha}^{0, T}(u, v)=\mathcal{E}^{0, T}(u, v)+\alpha(u, v)_{\mathcal{H}_{0, T}}$ and $(\cdot, \cdot)_{\mathcal{H}_{0, T}}$ denotes the usual inner product in $\mathcal{H}_{0, T}$.

In the paper by cap we denote the parabolic capacity determined by the form $\mathcal{E}$ (for the construction and properties of cap see [23, Sect. 4] or [24, Sect. 6.2]). We will say that some property is satisfied quasi-everywhere (q.e. for short) if it is satisfied except for some Borel subset of $E^{1}$ of capacity cap zero. Using cap we define quasi-continuity as in [23]. By [23, Theorem 4.1] each function $u \in \mathcal{W}$ has a quasi-continuous $m_{1}$ version, which we will denote by $\tilde{u}$.

Let $\mu$ be a Borel signed measure on $E^{1}$. In what follows $|\mu|$ stands for the total variation of $\mu$. By $\mathcal{M}_{0, b}\left(E^{1}\right)$ we denote the set of all Borel measures on $E^{1}$ such that $|\mu|$ does not charge sets of zero capacity cap and $|\mu|\left(E^{1}\right)<\infty$. By $\mathcal{M}_{0, b}\left(E_{0, T}\right)$ we denote the subset of $\mathcal{M}_{0, b}\left(E^{1}\right)$ consisting of all measures with support in $E_{0, T}$.

\subsection{Markov Processes}

By general results from the theory of Markov process (see, e.g., [24, Theorems 6.3.1, 6.3.10]) there exists a continuous Hunt process $\mathbf{M}=\left(\Omega,\left(\mathcal{F}_{t}\right)_{t \geq 0},\left(\mathbf{X}_{t}\right)_{t \geq 0}, \zeta\right.$, $\left.\left(P_{z}\right)_{z \in E^{1} \cup \Delta}\right)$ with state space $E^{1}$, life time $\zeta$ and cemetery state $\Delta$ properly associated with $\mathcal{E}$ in the resolvent sense. By [23, Theorem 5.1],

$$
\mathbf{X}_{t}=\left(\tau(t), X_{\tau(t)}\right), \quad t \geq 0,
$$

where $\tau(t)$ is the uniform motion to the right, i.e. $\tau(t)=\tau(0)+t, \tau(0)=s, P_{z}$-a.s. for $z=(s, x)$. For an alternative construction of $\mathbf{M}$, for which the starting point is the fundamental solution for the operator $\frac{\partial}{\partial t}+L_{t}$, see [11, Sect. 2]. It is also known (this follows for instance from the construction of $\mathbf{M}$ given in [11]) that $\mathbb{X}=\left\{\left(X, P_{s, x}\right)\right.$ : 
$\left.\left.(s, x) \in \mathbb{R}_{+} \times E\right)\right\}$, where $X$ is the second component of $\mathbf{X}$, is a continuous timeinhomogeneous Markov process whose transition density $p_{E}$ is the Green function for $\frac{\partial}{\partial t}+L_{t}$ on $[0, T) \times E$ (for construction and properties of Green's function see [1]).

Let $\mu, v$ be Borel measures on $E^{1}$ and $E$, respectively. In what follows we write $P_{\mu}(\cdot)=\int_{E^{1}} P_{s, x}(\cdot) d \mu(s, x), P_{s, v}(\cdot)=\int_{E} P_{s, x}(\cdot) v(d x)$. By $E_{\mu}$ (resp. $\left.E_{s, v}\right)$ we denote the expectation with respect to $P_{\mu}\left(\right.$ resp. $\left.P_{s, v}\right)$.

Note that if $u$ is quasi-continuous then it is M-quasi-continuous, i.e. for q.e. $(s, x) \in$ $E^{1}, P_{s, x}\left([0, \infty) \ni t \mapsto u\left(\mathbf{X}_{t}\right)\right.$ is continuous)=1 (see, e.g., [23, p. 298]).

Set $\sigma_{B}=\inf \left\{t>0, \mathbf{X}_{t} \in B\right\}$. It is known (see remarks following [23, (5.2)]) that for a Borel set $B \subset E^{1}, \operatorname{cap}(B)=0$ if and only if $B$ is $\mathbf{M}$-exceptional, i.e.

$$
P_{m_{1}}\left(\sigma_{B}<\infty\right)=\int_{\mathbb{R}} P_{s, m}\left(\exists t>0: \mathbf{X}_{t} \in B\right) d s=0 .
$$

Remark 2.1 (i) From (2.6) and the fact that $p_{E}(s, x, t, \cdot)$ is strictly positive on $E$ it follows that $\operatorname{cap}(\{s\} \times \Gamma)>0$ for any $s \in(0, T)$ and Borel set $\Gamma \subset E$ such that $m(\Gamma)>0$. Hence, if some property holds for q.e. $(s, x) \in(0, T) \times E$ then it holds for $m$-a.e. $x \in E$ for every $s \in(0, T)$.

(ii) If $P_{m_{1}}\left(\sigma_{B}<\infty\right)=0$ then in fact $P_{s, x}\left(\sigma_{B}<\infty\right)=0$ for q.e. $(s, x) \in E^{1}$. This follows from the fact that the function $(s, x) \mapsto P_{s, x}\left(\sigma_{B}<\infty\right)$ is excessive (see remark at the end of [23, p. 294]).

Let $A$ be a positive continuous additive functional of $\mathbf{M}$ and let $\mu \in \mathcal{M}_{0, b}\left(E_{0, T}\right)$ be a positive measure. We will say that $A$ corresponds to $\mu$ (or $\mu$ corresponds to $A$ ) if for q.e. $(s, x) \in[0, T) \times E$,

$$
E_{s, x} \int_{0}^{\zeta_{\tau}} f\left(\mathbf{X}_{t}\right) d A_{t}=\int_{s}^{T} \int_{E} f(t, y) p_{E}(s, x, t, y) \mu(d t d y)
$$

for every $f \in C_{c}([0, T] \times E)$, where

$$
\zeta_{\tau}=\zeta \wedge(T-\tau(0))
$$

Since $p_{E}(s, x, \cdot, \cdot)$ is strictly positive on $(s, T] \times E$, if $\mu_{1}$ and $\mu_{2}$ correspond to $A$ then $\mu_{1}=\mu_{2}$. It is also known (see, e.g., Proposition (2.12) in [3, Chap. IV]) that if $\mu \in \mathcal{M}_{0, b}\left(E_{0, T}\right)$ corresponds to $A^{1}$ and to $A^{2}$ then $A^{1}$ and $A^{2}$ are equivalent, i.e. for each $t \in[0, T], P_{s, x}\left(A_{t}^{1}=A_{t}^{2}\right)=1$ for q.e. $(s, x) \in[0, T) \times E$. If $\mu \in$ $\mathcal{M}_{0, b}\left(E_{0, T}\right)$ then we say that $\mu$ corresponds to $A$ if $A=A^{+}-A^{-}$, where $A^{+}, A^{-}$ are positive continuous additive functionals of $\mathbf{M}$ such that $A^{+}$corresponds to $\mu^{+}$ and $A^{-}$corresponds to $\mu^{-}$(here $\mu^{+}$(resp. $\mu^{-}$) is the positive (resp. negative) part of the Jordan decomposition of $\mu$ ). Also note that (2.7) is some sort of the Revuz correspondence.

The following proposition is probably well known, but we do not have a reference. 
Proposition 2.2 Let $\varphi \in H, f \in L^{2}(0, T ; H)$ and let $\tilde{u}$ be a quasi-continuous version of the solution $u \in[\mathcal{W}]^{m}$ of the Cauchy problem

$$
\frac{\partial u}{\partial t}+L_{t} u=-f, \quad u(T)=\varphi .
$$

Then

$$
\tilde{u}\left(\mathbf{X}_{t \wedge \zeta_{\tau}}\right)-\tilde{u}\left(\mathbf{X}_{0}\right)=M_{t \wedge \zeta_{\tau}}^{[u]}-\int_{0}^{t \wedge \zeta_{\tau}} f\left(\mathbf{X}_{\theta}\right) d \theta, \quad t \in[0, T],
$$

where $M^{[u]}=\left(M^{[u], 1}, \ldots, M^{[u], m}\right)$ is a continuous martingale AF of $\mathbf{M}$ with the quadratic variation

$$
\left[M^{[u], k}\right]_{t}=\sum_{i, j=1}^{d} \int_{0}^{t} a_{i j} \frac{\partial u^{k}}{\partial x_{i}} \frac{\partial u^{k}}{\partial x_{j}}\left(\mathbf{X}_{\theta}\right) d \theta, \quad t \geq 0, \quad k=1, \ldots, m .
$$

Moreover,

$$
M_{t}^{[u], k}=\sum_{i=1}^{d} \int_{0}^{t} \frac{\partial u^{k}}{\partial x_{i}}\left(\mathbf{X}_{\theta}\right) d M_{\theta}^{i}, \quad t \geq 0,
$$

where $M=\left(M^{1}, \ldots, M^{d}\right)$ is a continuous martingale $A F$ of $\mathbf{M}$ with the quadratic covariation

$$
\left[M^{i}, M^{j}\right]_{t}=\sum_{i, j=1}^{d} \int_{0}^{t} a_{i j}\left(\mathbf{X}_{\theta}\right) d \theta, \quad t \geq 0, \quad k=1, \ldots, m
$$

Proof We provide sketch of the proof. Since $\mathcal{E}^{0, T}$ is a generalized Dirichlet form, it follows from [29, Theorem 4.5] that

$$
\tilde{u}\left(\mathbf{X}_{t \wedge \zeta_{\tau}}\right)-\tilde{u}\left(\mathbf{X}_{0}\right)=M_{t \wedge \zeta_{\tau}}^{[u]}+N_{t \wedge \zeta_{\tau}}^{[u]}, \quad t \in[0, T],
$$

where $M^{[u]}=\left(M^{[u], 1}, \ldots, M^{[u], m}\right)$ is a continuous martingale AF of $\mathbf{M}$ and $N^{[u]}$ is continuous AF of $\mathbf{M}$ of finite energy. Let $A_{t}=\int_{0}^{t} f\left(\mathbf{X}_{\theta}\right) d \theta, t \in[0, T]$. One can show that for every $v \in \mathcal{W}_{T}$,

$$
\lim _{\alpha \rightarrow \infty} \alpha^{2} E_{v \cdot m_{1}} \int_{0}^{\zeta_{\tau}} e^{-\alpha t} A_{t} d t=(f, v)_{L^{2}(0, T ; H)}
$$

Since $u$ satisfies (2.8), we see that the left-hand side of (2.12) equals $\mathcal{E}^{0, T}(u, v)$. From this and an analogue of [23, Theorem 7.4] for the form $\mathcal{E}^{0, T}$ it follows that $N_{t}^{[u]}=-A_{t}$, $t \in[0, T]$. Modifying slightly the proof of [23, (7.13)] we show (2.9). Finally, to show (2.10), let us denote by $M^{u, k}$ the process on the right-hand side of (2.10) and consider 
a sequence $\left\{u_{n}\right\}$ of smooth functions such that $u_{n} \rightarrow u$ in $\mathcal{W}$. Then by the chain rule (see [29, Theorem 5.5]), $M^{\left[u_{n}\right], k}=M^{u_{n}, k}$. It is clear that $\left\{M^{u_{n}, k}\right\}_{n}$ is $e$-convergent to $M^{u, k}$. On the other hand, arguing as in the proof of [23, Theorem 7.2]) we show that $\left\{M^{\left[u_{n}\right], k}\right\}_{n}$ is $e$-convergent to $M^{[u], k}$, which proves (2.10).

Let $Y$ be a special $\left(\left(\mathcal{F}_{t}\right), P_{z}\right)$-semimartingale on $[0, T]$, i.e. an $\left(\left(\mathcal{F}_{t}\right), P_{z}\right)$-semimartingale admitting a (unique) decomposition $Y_{t}=Y_{0}+M_{t}+B_{t}, t \in[0, T]$, with $M_{0}=B_{0}=0, M$ a local $\left(\left(\mathcal{F}_{t}\right), P_{z}\right)$-martingale and $B$ an $\left(\mathcal{F}_{t}\right)$-predictable finite variation process (see, e.g., [27, Sect. III.7]). Recall that the $\mathcal{H}^{2}\left(P_{z}\right)$ norm of $Y$ is defined to be $\|Y\|_{\mathcal{H}^{2}\left(P_{z}\right)}=\left(E_{z}\left|Y_{0}\right|^{2}\right)^{1 / 2}+\left(E_{z}[M]_{T}\right)^{1 / 2}+\left(E_{z}|B|_{T}^{2}\right)^{1 / 2}$, where $[M]$ is the quadratic variation of $M$ and $|B|_{T}$ is the variation of $B$ on the interval $[0, T]$. By $\mathcal{H}^{2}\left(P_{z}\right)$ we denote the space of all special $\left(\left(\mathcal{F}_{t}\right), P_{z}\right)$-semimartingales on $[0, T]$ with finite $\mathcal{H}^{2}\left(P_{z}\right)$ norm.

Remark 2.3 (i) Let $z \in E_{0, T}$ and let $\varphi, f$ satisfy the assumptions of Proposition 2.2. Then $M^{[u]}$ of Proposition 2.2 is a martingale under $P_{z}$ (see [29, p. 327]), $E_{z}\left|\tilde{u}\left(\mathbf{X}_{0}\right)\right|^{2}=|\tilde{u}(z)|^{2}<\infty, E_{z}\left[M_{\cdot \wedge \zeta_{\tau}}^{[u], k}\right]_{T}<\infty, E_{z}\left(\int_{0}^{\zeta_{\tau}}\left|f\left(\mathbf{X}_{t}\right)\right| d t\right)^{2}<\infty$ for q.e. $z \in E_{0, T}$. Hence $Y=\tilde{u}\left(\mathbf{X}_{\cdot \wedge \zeta_{\tau}}\right)$ is a semimartingale of class $\mathcal{H}^{2}\left(P_{z}\right)$ for q.e. $z \in E_{0, T}$.

(ii) Let $M=\left(M^{1}, \ldots, M^{d}\right)$ be the AF of Proposition 2.2. Then $M$ is a martingale under $P_{z}$ for q.e. $z \in E_{0, T}$ (see [24, Sect. 5.1.2]). Set

$$
B_{t}^{i}=\sum_{j=1}^{d} \int_{0}^{t} \sigma_{i j}^{-1}\left(\mathbf{X}_{\theta}\right) d M_{\theta}^{j}, \quad t \geq 0, \quad i=1, \ldots, d
$$

where $\sigma^{-1}$ is the inverse matrix of $\sigma$. By Lévy's theorem and (2.11), for q.e. $z \in E_{0, T}$ the process $B=\left(B^{1}, \ldots, B^{d}\right)$ is under $P_{z}$ a $d$-dimensional standard Brownian motion with respect to $\left(\mathcal{F}_{t}\right)_{t \geq 0}$. Finally, note that by (2.10) and (2.13),

$$
M_{t}^{[u], k}=\sum_{i, j=1}^{d} \int_{0}^{t} \frac{\partial u^{k}}{\partial x_{i}}\left(\mathbf{X}_{\theta}\right) \sigma_{i j}\left(\mathbf{X}_{\theta}\right) d B_{\theta}^{j}, \quad t \geq 0, \quad k=1, \ldots, m .
$$

\section{Probabilistic Solutions of the Obstacle Problem}

Let $\varphi: E \rightarrow \mathbb{R}^{m}, f: E_{T} \times \mathbb{R}^{m} \times \mathbb{R}^{m \times d} \rightarrow \mathbb{R}^{m}$ be measurable functions and let $D=\left\{D(t, x):(t, x) \in E_{T}\right\}$ be a family of closed convex sets in $\mathbb{R}^{m}$ with nonempty interiors. Given $\varepsilon>0$ we set $D^{*}(t, x)=\{y \in D(t, x): \operatorname{dist}(y, \partial D(t, x)) \geq \varepsilon\}$. We will assume that

(A1) $\varphi(x) \in D(T, x)$ for $x \in E, \varphi \in H$,

(A2) $f(\cdot, \cdot, 0,0) \in L^{2}(0, T ; H)$,

(A3) $f: E_{T} \times \mathbb{R}^{m} \times \mathbb{R}^{m \times d} \rightarrow \mathbb{R}^{m}$ is a measurable function and there exist $\alpha, \beta \geq 0$ such that

$$
\left|f\left(t, x, y_{1}, z_{1}\right)-f\left(t, x, y_{2}, z_{2}\right)\right| \leq \alpha\left|y_{1}-y_{2}\right|+\beta\left\|z_{1}-z_{2}\right\|
$$


for all $y_{1}, y_{2} \in \mathbb{R}^{m}$ and $z_{1}, z_{2} \in \mathbb{R}^{m \times d}$.

As for the family $D$, we will need the following assumptions:

(D1) The sets $D(t, x)$ are bounded uniformly in $(t, x) \in E_{T}$ and the mapping $E_{T} \ni$ $(t, x) \mapsto D(t, x) \in$ Conv is continuous.

(D2) For some $\varepsilon>0$ chosen so that $\operatorname{Int} D^{*}(t, x) \neq \varnothing$ for $(t, x) \in E_{T}$ and some $f_{*} \in L^{2}(0, T ; H)$ and $\varphi_{*} \in H$ such that $\varphi_{*}(x) \in D^{*}(T, x)$ for $x \in E$ there exists a solution $u_{*} \in \mathcal{W}$ of the Cauchy problem (1.5) such that $u_{*}(t, x) \in D^{*}(t, x)$ for q.e. $(t, x) \in E_{0, T}$.

Remark 3.1 Condition (D2) implies the following condition:

(D3) There exists a quasi-continuous function $u_{*}: E_{0, T} \rightarrow \mathbb{R}^{m}$ such that the process $Y^{*}$ defined as $Y_{t}^{*}=u_{*}\left(\mathbf{X}_{t \wedge \zeta_{\tau}}\right), t \in[0, T]$, has the following properties:

(a) $Y_{t}^{*} \in \operatorname{Int} D\left(\mathbf{X}_{t \wedge \zeta_{\tau}}\right)$ and $\operatorname{dist}\left(Y_{t}^{*}, \partial D\left(\mathbf{X}_{t \wedge \zeta_{\tau}}\right)\right) \geq \varepsilon, t \in[0, T], P_{s, x}$-a.s. for q.e. $(s, x) \in E_{0, T}$,

(b) $Y^{*, i}, i=1, \ldots, m$, is a special semimartingale of class $\mathcal{H}^{2}\left(P_{s, x}\right)$ for q.e. $(s, x) \in[0, T) \times E$.

To see this, it suffices to consider a quasi-continuous version $\tilde{u}_{*}$ of $u_{*}$ of condition (D2). From quasi-continuity of $\tilde{u}_{*}$, condition (D2) and Remark 3.5 it follows that the process $Y^{*}=\tilde{u}_{*}\left(\mathbf{X}_{\wedge \wedge \zeta_{\tau}}\right)$ satisfies condition (a) [see Remark 3.5(i) below]. By Proposition 2.2, $Y^{*}$ admits decomposition (2.8) with $u$ replaced by $u_{*}$ and $f$ replaced by $f_{*}$. Therefore $Y^{*}$ satisfies condition (b) by remarks following Proposition 2.3 .

Condition (D2) is satisfied in the following natural situations.

Example 3.2 Assume that one can find $r>0$ such that for every $(t, x) \in E_{T}$, $B(0, r) \subset D(t, x)$, where $B(0, r)$ denotes the open ball with radius $r$ and center at 0 . Then $D$ satisfies (D2). To see this, it suffices to consider the constant function $u_{*}=\left(u_{*}^{1}, \ldots, u_{*}^{m}\right)=(0, \ldots, 0)$. For instance, the above condition on $D$ is satisfied if $D(t, x)=\left\{y \in \mathbb{R}^{m}: h^{i}(t, x) \leq y_{i} \leq \bar{h}^{i}(t, x), i=1, \ldots, m\right\}$, where $\underline{h}^{i}, \bar{h}^{i}:[0, T] \times \bar{E} \rightarrow \mathbb{R}$ are continuous functions such that $\underline{h}^{i}(t, x)<0<\bar{h}^{i}(t, x)$ for $(t, x) \in[0, T] \times \bar{E}, i=1, \ldots, m$.

Example 3.3 Let $D(t, x)=\left\{y \in \mathbb{R}^{m}: \underline{h}^{i}(t, x) \leq y_{i} \leq \bar{h}^{i}(t, x), i=1, \ldots, m\right\}$, where $\underline{h}^{i}, \bar{h}^{i}:[0, T] \times \bar{E} \rightarrow \mathbb{R}$ are continuous functions such that $\underline{h}^{i}(t, x)<\bar{h}^{i}(t, x)$ for $(t, \bar{x}) \in[0, T] \times \bar{E}$. Assume that there are continuous functions $\underline{h}_{0}^{i}, \bar{h}_{0}^{i}:[0, T] \times$ $\bar{E} \rightarrow \mathbb{R}$ such that $\underline{h}^{i}(t, x) \leq \underline{h}_{0}^{i}(t, x)<\bar{h}_{0}^{i}(t, x) \leq \bar{h}^{i}(t, x)$ for $(t, x) \in[0, T] \times \bar{E}$, $\underline{h}_{0}^{i}, \bar{h}_{0}^{i} \in \mathcal{W}_{0, T}$ and $\left(\frac{\partial}{\partial t}+L_{t}\right) \underline{h}_{0}^{i},\left(\frac{\partial}{\partial t}+L_{t}\right) \bar{h}_{0}^{i} \in L^{2}\left(0, T ; L^{2}(E)\right), i=1, \ldots, m$. Then (D2) is satisfied with $u_{*}^{i}=\left(\bar{h}_{0}^{i}+\underline{h}_{0}^{i}\right) / 2, i=1, \ldots, m$. Of course, the last condition for $\underline{h}_{0}^{i}, \bar{h}_{0}^{i}$ is satisfied if $\underline{h}_{0}^{i}, \bar{h}_{0}^{i} \in W_{2}^{1,2}((0, T) \times E)$ and $\frac{\partial a_{i j}}{\partial x_{k}} \in L^{\infty}((0, T) \times E)$ for $i, j, k=1, \ldots, d$.

In what follows we are going to show that under assumptions (A1)-(A3), (D1), (D2) there exists a unique solution of the problem (1.1), (1.2). It is convenient to start with probabilistic solutions. Solutions of (1.1), (1.2) in the sense of the definition given in Sect. 1 will be studied in the next section. Note that the definition formulated below is an extension, to the case of systems, of the probabilistic definition adopted in $[9,11]$ in case of single equation. 
Definition 3.4 We say that a pair $(u, \mu)$ consisting of a measurable function $u=$ $\left(u^{1}, \ldots, u^{m}\right): E_{0, T} \rightarrow \mathbb{R}^{m}$ and a measure $\mu=\left(\mu^{1}, \ldots, \mu^{m}\right)$ on $E_{0, T}$ is a probabilistic solution of the obstacle problem with data $\varphi, f, D(\operatorname{OP}(\varphi, f, D)$ for short) if

(a) $u$ is quasi-continuous, $f_{u}^{i} \in L^{1}\left(E_{0, T}\right), \mu^{i} \in \mathcal{M}_{0, b}\left(E_{0, T}\right), i=1, \ldots, m$,

(b) For q.e. $(s, x) \in E_{0, T}$ and $i=1, \ldots, m$,

$$
\begin{aligned}
u^{i}\left(\mathbf{X}_{t}\right)= & \varphi^{i}\left(\mathbf{X}_{\zeta_{\tau}}\right)+\int_{t \wedge \zeta_{\tau}}^{\zeta_{\tau}} f_{u}^{i}\left(\mathbf{X}_{\theta}\right) d \theta+\int_{t \wedge \zeta_{\tau}}^{\zeta_{\tau}} d A_{\theta}^{\mu^{i}} \\
& -\int_{t \wedge \zeta_{\tau}}^{\zeta_{\tau}} \sigma \nabla u^{i}\left(\mathbf{X}_{\theta}\right) d B_{\theta}, \quad t \in[0, T], \quad P_{S, x} \text {-a.s. }
\end{aligned}
$$

where $A^{\mu^{i}}, i=1, \ldots, m$, is the continuous additive functional of $\mathbf{M}$ associated with $\mu^{i}$ in the sense of (2.7),

(c) $u(t, x) \in D(t, x)$ for q.e. $(t, x) \in E_{0, T}$ and for every quasi-continuous function $h=\left(h^{1}, \ldots, h^{m}\right)$ such that $h(t, x) \in D(t, x)$ for q.e. $(t, x) \in E_{0, T}$ we have

$$
\int_{0}^{\zeta_{\tau}}\left\langle u\left(\mathbf{X}_{t}\right)-h\left(\mathbf{X}_{t}\right), d A_{t}^{\mu}\right\rangle \leq 0, \quad P_{s, x} \text {-a.s. }
$$

for q.e. $(s, x) \in E_{T}$.

Remark 3.5 (i) From the fact that $u$ is quasi-continuous and $u(t, x) \in D(t, x)$ for q.e. $(t, x) \in E_{0, T}$ it follows that $u\left(\mathbf{X}_{t \wedge \zeta_{\tau}}\right) \in D\left(\mathbf{X}_{t \wedge \zeta_{\tau}}\right), t \in[0, T], P_{s, x}$-a.s. for q.e. $(s, x) \in E_{0, T}$. To see this, let us set $B=\left\{(t, x) \in E_{0, T}: u(t, x) \notin D(t, x)\right\}$ and $\sigma_{B}=\inf \left\{t>0: \mathbf{X}_{t} \in B\right\}$. Since $\operatorname{cap}(B)=0$, the set $B$ is M-exceptional, and hence, by 2.1(ii), $P_{s, x}\left(\sigma_{B}<\infty\right)=0$ for q.e. $(s, x) \in E_{0, T}$. Hence $P_{s, x}\left(\mathbf{X}_{t \wedge \zeta_{\tau}} \in\right.$ $B, t \in(0, T])=0$, which implies that $u\left(\mathbf{X}_{t \wedge \zeta_{\tau}}\right) \in D\left(\mathbf{X}_{t \wedge \zeta_{\tau}}\right), t \in(0, T], P_{s, x^{-}}$ a.s. for q.e. $(s, x) \in E_{0, T}$. In fact, we can replace $(0, T]$ by $[0, T]$, because $\mathbf{X}$ is right-continuous at $t=0$ and $D$ satisfies (D1).

(ii) Conditions (3.1), (3.2) of the above definition say that under $P_{s, x}$ the pair $\left(Y_{t}, Z_{t}\right)=\left(u\left(\mathbf{X}_{t \wedge \zeta_{\tau}}\right), \sigma \nabla u\left(\mathbf{X}_{t \wedge \zeta_{\tau}}\right)\right), t \in[0, T]$, is a solution of the generalized Markov-type reflected BSDE with final condition $\varphi$, coefficient $f$, finite variation process $A^{\mu}$ and obstacle $D$.

(iii) Taking $t=0$ in (3.1) and then integrating with respect to $P_{s, x}$ we see that for q.e. $(s, x) \in E_{0, T}$,

$$
u^{i}(s, x)=E_{s, x}\left(\varphi^{i}\left(\mathbf{X}_{\zeta_{\tau}}\right)+\int_{0}^{\zeta_{\tau}} f_{u}^{i}\left(\mathbf{X}_{\theta}\right) d \theta+\int_{0}^{\zeta_{\tau}} d A_{\theta}^{\mu^{i}}\right), \quad i=1, \ldots, m .
$$

By this and [10, Proposition 3.4], if $u \in L^{2}(0, T ; H)$ then $u^{i} \in C\left([0, T] ; L^{2}(E)\right)$, $i=1, \ldots, m$.

(iv) From continuity of $A^{\mu^{i}}$ and (2.7) one can deduce that $\mu^{i}(\{t\} \times E)=0$ for every $t \in[0, T]$. 
We begin with uniqueness of probabilistic solutions.

Proposition 3.6 Assume (A3), (D1). Then there exists at most one probabilistic solution of $\mathrm{OP}(\varphi, f, D)$.

Proof Let $\left(u_{1}, \mu_{1}\right),\left(u_{2}, \mu_{2}\right)$ be two solutions of $\operatorname{OP}(\varphi, f, \mathcal{D})$. Set $u=u_{1}-u_{2}$, $\mu=\mu_{1}-\mu_{2}$ and $Y_{t}=u\left(\mathbf{X}_{t \wedge \zeta_{\tau}}\right), Z_{t}=\sigma \nabla u\left(\mathbf{X}_{t \wedge \zeta_{\tau}}\right), K_{t}=A_{t \wedge \zeta_{\tau}}^{\mu}, D_{t}=D\left(\mathbf{X}_{t \wedge \zeta_{\tau}}\right)$, $t \in[0, T]$. Applying Itô's formula and using (A3) shows that there is $C>0$ depending only on $\alpha, \beta$ such that for q.e. $(s, x) \in E_{T}$,

$$
E_{s, x}\left|Y_{t}\right|^{2}+\frac{1}{2} E_{s, x} \int_{t \wedge \zeta_{\tau}}^{\zeta_{\tau}}\left\|Z_{\theta}\right\|^{2} d \theta \leq C E_{s, x} \int_{t \wedge \zeta_{\tau}}^{\zeta_{\tau}}\left|Y_{\theta}\right|^{2} d \theta+2 E_{s, x} \int_{t \wedge \zeta_{\tau}}^{\zeta_{\tau}}\left\langle Y_{\theta}, d K_{\theta}\right\rangle
$$

for all $t \in[s, T]$. By (D1) and (2.3) the mapping $E_{T} \times \mathbb{R}^{m} \ni(t, x, y) \mapsto \Pi_{D(t, x)}(y) \in$ $\mathbb{R}^{m}$ is continuous. Therefore the mappings $E_{T} \ni(t, x) \mapsto \Pi_{D(t, x)}\left(u_{i}(t, x)\right) \in \mathbb{R}^{m}$, $i=1,2$, are quasi-continuous. Using this and condition (3.2) we get

$$
\begin{aligned}
&\left\langle Y_{t}, d K_{t}\right\rangle=\left\langle Y_{t}^{1}-\Pi_{D_{t}}\left(Y_{t}^{2}\right), d K_{t}^{1}\right\rangle+\left\langle\Pi_{D_{t}}\left(Y_{t}^{2}\right)-Y_{t}^{2}, d K_{t}^{1}\right\rangle \\
&+\left\langle Y_{t}^{2}-\Pi_{D_{t}}\left(Y_{t}^{1}\right), d K_{t}^{2}\right\rangle+\left\langle\Pi_{D_{t}}\left(Y_{t}^{1}\right)-Y_{t}^{1}, d K_{t}^{2}\right\rangle \\
& \leq\left|\Pi_{D_{t}}\left(Y_{t}^{2}\right)-Y_{t}^{2}\right| d\left|K^{1}\right|_{t}+\left|\Pi_{D_{t}}\left(Y_{t}^{1}\right)-Y_{t}^{1}\right| d\left|K^{2}\right|_{t}=0 .
\end{aligned}
$$

Hence

$$
E_{s, x}\left|Y_{t}\right|^{2}+\frac{1}{2} E_{s, x} \int_{t \wedge \zeta_{\tau}}^{\zeta_{\tau}}\left\|Z_{\theta}\right\|^{2} d \theta \leq C \int_{t \wedge \zeta_{\tau}}^{\zeta_{\tau}} E_{s, x}\left|Y_{\theta}\right|^{2} d \theta, \quad t \in[s, T]
$$

for q.e. $(s, x) \in E_{T}$. Applying Gronwall's lemma yields $E_{s, x}\left|Y_{t}\right|^{2}=0$. Since $Y^{1}, Y^{2}$ are continuous, $Y_{t}^{1}=Y_{t}^{2}, t \in[0, T], P_{s, x}$-a.s. for q.e. $(s, x) \in E_{T}$. This and (3.3) imply that $Z^{1}=Z^{2}, P_{s, x} \otimes d t$-a.e. on $\Omega \times[0, T]$. That $K_{t}^{1}=K_{t}^{2}, t \in[0, T], P_{s, x}$-a.s. now follows from the fact that $\left(Y^{i}, Z^{i}, K^{i}\right), i=1,2$ satisfy the equation of condition (b) of Definition 3.4.

Proposition 3.7 Assume that $\varphi, f$ satisfy(A1)-(A3), D satisfies (D1) and there exists $u_{*}=\left(u_{*}^{1}, \ldots u_{*}^{m}\right) \in[\mathcal{W}]^{m}$ such that $u_{*} \in D(t, x)$ for q.e. $(t, x) \in E_{0, T}$.

(i) For every $n \in \mathbb{N}$ there exists a unique strong solution $u_{n} \in[\mathcal{W}]^{m}$ of the problem

$$
\frac{\partial u_{n}}{\partial t}+L_{t} u_{n}=-f_{u_{n}}+n\left(u_{n}-\Pi_{D(\cdot, \cdot)}\left(u_{n}\right)\right), \quad u_{n}(T)=\varphi .
$$

(ii) There is $C$ depending only on $\alpha, \beta, \Lambda$ and $\left\|u_{*}\right\|_{[\mathcal{W}]^{m}}$ such that

$$
\sup _{0 \leq s \leq T}\left\|u_{n}(s)\right\|_{H}^{2}+\int_{0}^{T}\left\|\nabla u_{n}(t)\right\|_{H}^{2} d t \leq C\left(\|\varphi\|_{H}^{2}+\|f(\cdot, \cdot, 0,0)\|_{L^{2}(0, T ; H)}^{2}\right) .
$$


Proof Since $u$ is a strong solution of (3.4) if and only if $\hat{u}=e^{\lambda t} u$ is a strong solution of (3.4) with $L_{t}$ replaced by $L_{t}-\lambda, \varphi$ replaced by $e^{\lambda T} \varphi \in H$ and $f$ replaced by some $\hat{f}$ still satisfying (A2) and (A3), without loss of generality we may replace $L_{t}$ in (3.4) by $L_{t}-\lambda$. Let $\mathcal{A}: L^{2}(0, T ; V) \rightarrow L^{2}\left(0, T ; V^{\prime}\right)$ be the operator defined as $(\mathcal{A} v)(t)=L_{t} v(t)-\lambda v(t)+f_{v(t)}-n\left(v(t)-\Pi_{D(\cdot, \cdot)}(v(t))\right)$. By (D1) and (2.3) the mapping $(t, x, y) \mapsto-n\left(y-\Pi_{D(t, x)}(y)\right)$ is continuous and Lipschitz continuous in $y$ for each fixed $(t, x) \in E_{T}$. Therefore for sufficiently large $\lambda>0$ (depending on $\alpha$ and $\beta$ ) the operator $\mathcal{A}$ is bounded, hemicontinuous, monotone and coercive, i.e. satisfies condition (7.84) from [18, Chap. 2]. Therefore the existence of a unique strong solution $u_{n} \in[\mathcal{W}]^{m}$ of (3.4) follows from Theorem 7.1. and Remark 7.12 in [18, Chap. 2]. To prove (ii), set $\varphi_{*}=u_{*}(T), f_{*}=-\frac{\partial u_{*}}{\partial t}-L_{t} u_{*}$. Then $\varphi_{*} \in H, f_{*} \in L^{2}\left(0, T ; V^{\prime}\right)$, $u_{n}-u_{*} \in[\mathcal{W}]^{m},\left(u_{n}-u_{*}\right)(T)=\varphi-\varphi^{*}$ and

$$
\frac{\partial\left(u_{n}-u_{*}\right)}{\partial t}+L_{t}\left(u_{n}-u_{*}\right)=-\left(f_{u_{n}}-f_{*}\right)+n\left(u_{n}-\Pi_{D(\cdot, \cdot)}\left(u_{n}\right)\right) .
$$

Multiplying the above equation by $u_{n}-u_{*}$ and integrating by parts we obtain

$$
\begin{aligned}
\int_{s}^{T} & \left\langle\frac{\partial\left(u_{n}-u_{*}\right)}{\partial t}(t), u_{n}(t)-u_{*}(t)\right\rangle_{V^{\prime}, V} d t \\
- & \sum_{k=1}^{m} \int_{s}^{T} a\left(t ; u_{n}^{k}(t)-u_{*}^{k}(t), u_{n}^{k}(t)-u_{*}^{k}(t)\right) d t \\
= & -\int_{s}^{T}\left\langle f_{u_{n}}(t)-f_{*}(t), u_{n}(t)-u_{*}(t)\right\rangle_{V^{\prime}, V} d t \\
& +n \int_{S}^{T}\left(u_{n}(t)-\Pi_{D(\cdot, \cdot)}\left(u_{n}\right)(t), u_{n}(t)-u_{*}(t)\right)_{H} d t
\end{aligned}
$$

By (2.2), $\left(u_{n}(t)-\Pi_{D(\cdot, \cdot)}\left(u_{n}\right)(t), u_{n}(t)-u_{*}(t)\right)_{H} \geq 0$. Therefore from the above equality and (A3) it follows that

$$
\begin{aligned}
\frac{1}{2} \| & \left(u_{n}-u_{*}\right)(s) \|_{H}^{2}+\sum_{k=1}^{m} \int_{S}^{T} a\left(t ; u_{n}^{k}(t)-u_{*}^{k}(t), u_{n}^{k}(t)-u_{*}^{k}(t)\right) d t \\
\leq & \frac{1}{2}\left\|\varphi-\varphi_{*}\right\|_{H}^{2}+\int_{s}^{T}\left\|f_{*}(t)\right\|_{V^{\prime}} \cdot\left\|u_{n}(t)-u_{*}(t)\right\|_{V} d t \\
& +\int_{S}^{T}\left|\left(f(t, \cdot, 0,0), u_{n}(t)-u_{*}(t)\right)_{H}\right| d t \\
& +\int_{S}^{T}\left(\alpha\left\|u_{n}(t)\right\|_{H} \cdot\left\|u_{n}(t)-u_{*}(t)\right\|_{H}+\beta\left\|\nabla u_{n}(t)\right\|_{H}^{2} \cdot\left\|u_{n}(t)-u_{*}(t)\right\|_{H}^{2}\right) d t
\end{aligned}
$$

Using this and standard arguments (we apply Poincaré's inequality and Gronwall's lemma) shows (ii).

In the proof of our main theorem on existence and approximation we will use some additional notation. Let $\hat{\mathbf{M}}=\left(\hat{\mathbf{X}},\left(\hat{P}_{z}\right)_{z \in E^{1} \cup \Delta}\right)$ denote a dual process associated with 
the form defined by (2.4) (see [23, Theorem 5.1]). For $\mu \in \mathcal{M}_{0, b}\left(E^{1}\right)$ let $A^{\mu}$ denote the additive functional of $\mathbf{M}$ associated with $\mathbf{M}$ in the sense of (2.7), and let $\hat{A}^{\mu}$ denote the additive functional of $\hat{\mathbf{M}}$ associated with $\mu$. Given $\alpha \geq 0$ and $\mu \in \mathcal{M}_{0, b}\left(E^{1}\right)$ we set (whenever the integral exists)

$$
R_{\alpha}^{0, T} \mu(s, x)=E_{s, x} \int_{0}^{\zeta_{\tau}} e^{-\alpha t} d A_{t}^{\mu}, \quad \hat{R}_{\alpha}^{0, T} \mu(s, x)=\hat{E}_{s, x} \int_{0}^{\hat{\zeta} \wedge \tau(0)} e^{-\alpha t} d \hat{A}_{t}^{\mu}
$$

for $(s, x) \in E^{1}$, where $\hat{E}_{s, x}$ denotes the expectation with respect to $\hat{P}_{s, x}$ and $\hat{\zeta}$ is the life time of $\hat{\mathbf{M}}$. By $\hat{S}_{00}\left(E_{0, T}\right)$ we denote the set of all $\mu \in \mathcal{M}_{0, b}\left(E_{0, T}\right)$ such that $|\mu|$ is a finite energy integral measure on $E^{1}$ (see [23] for the definition) and $\left\|\hat{R}_{0}^{0, T}|\mu|\right\|_{\infty}<\infty$.

Theorem 3.8 Assume that $\varphi$, $f$ satisfy (A1)-(A3) and D satisfies (D1), (D2).

(i) There exists a unique solution $(u, \mu)$ of $\mathrm{OP}(\varphi, f, D)$.

(ii) $u^{i} \in C([0, T] ; H) \cap L^{2}\left(0, T ; H_{0}^{1}(E)\right), \mu^{i} \in \mathcal{M}_{0, b}\left(E_{0, T}\right), i=1, \ldots, m$, and

$$
\sup _{0<s \leq T}\|u(s)\|_{H}^{2}+\int_{0}^{T}\|\nabla u(t)\|_{H}^{2} d t \leq C\left(\|\varphi\|_{H}^{2}+\|f(\cdot, \cdot, 0,0)\|_{L^{2}(0, T ; H)}^{2}\right)
$$

with constant $C$ of Proposition 3.7.

(iii) Let $u_{n} \in[\mathcal{W}]^{m}$ be a solution of (3.4). Then

$$
\left\|u_{n}-u\right\|_{L^{2}(0, T ; H)}^{2} \rightarrow 0, \quad \int_{0}^{T}\left\|\nabla\left(u_{n}-u\right)(t)\right\|_{H}^{2} d t \rightarrow 0
$$

and

$$
\mu_{n} \rightarrow \mu \text { weakly }^{*} \text { on }(0, T] \times E \text {. }
$$

Proof Let $u_{n} \in[\mathcal{W}]^{m}$ be a quasi-continuous version of a strong solution of (3.4). Since $-f_{u_{n}}+n\left(u_{n}-\Pi_{D(\cdot, \cdot)}\left(u_{n}\right)\right) \in L^{2}(0, T ; H)$, it follows from Proposition 2.2 (and remark following it) that for q.e. $(s, x) \in E_{0, T}$ the pair

$$
\left(Y_{t}^{n}, Z_{t}^{n}\right)=\left(u_{n}\left(\mathbf{X}_{t \wedge \zeta_{\tau}}\right), \sigma \nabla u_{n}\left(\mathbf{X}_{t \wedge \zeta_{\tau}}\right)\right), \quad t \in[0, T]
$$

is a solution of the BSDE

$$
\begin{aligned}
u_{n}\left(\mathbf{X}_{t \wedge \zeta_{\tau}}\right)= & \varphi\left(\mathbf{X}_{\zeta_{\tau}}\right)+\int_{t \wedge \zeta_{\tau}}^{\zeta_{\tau}}\left(f\left(\mathbf{X}_{\theta}, u_{n}\left(\mathbf{X}_{\theta}\right), \sigma \nabla u_{n}\left(\mathbf{X}_{\theta}\right)\right) d \theta\right. \\
& +\int_{t \wedge \zeta_{\tau}}^{\zeta_{\tau}} K_{\theta}^{n}-\int_{t \wedge \zeta_{\tau}}^{\zeta_{\tau}} \sigma \nabla u_{n}\left(\mathbf{X}_{\theta}\right) d B_{\theta}, \quad t \in[0, T], \quad P_{s, x} \text {-a.s., }
\end{aligned}
$$


where

$$
K_{t}^{n}=-n \int_{t \wedge \zeta_{\tau}}^{\zeta_{\tau}}\left(u_{n}\left(\mathbf{X}_{\theta}\right)-\Pi_{D\left(\mathbf{X}_{\theta}\right)}\left(u_{n}\left(\mathbf{X}_{\theta}\right)\right)\right) d \theta
$$

Set

$$
\left(Y_{t}^{*}, Z_{t}^{*}\right)=\left(u_{*}\left(\mathbf{X}_{t \wedge \zeta_{\tau}}\right), \sigma \nabla u_{*}\left(\mathbf{X}_{t \wedge \zeta_{\tau}}\right)\right), \quad t \in[0, T],
$$

where $u_{*}$ denotes a quasi-continuous version of the function from condition (D2). By Remark 3.1, $Y^{*}$ satisfies (D3). By Itô's formula,

$$
\begin{gathered}
E_{s, x}\left(\left|Y_{t}^{n}-Y_{t}^{*}\right|^{2}+\int_{t}^{\zeta_{\tau}}\left\|Z_{\theta}^{n}-\sigma \nabla u_{*}\left(\mathbf{X}_{\theta}\right)\right\|^{2} d \theta\right) \leq E_{S, x}\left|\left(\varphi-\varphi_{*}\right)\left(\mathbf{X}_{\zeta_{\tau}}\right)\right|^{2} \\
\quad+2 E_{S, x}\left(\int_{t}^{\zeta_{\tau}}\left\langle Y_{\theta}^{n}-Y_{\theta}^{*},\left(f_{u_{n}}-f_{*}\right)\left(\mathbf{X}_{\theta}\right)\right\rangle d \theta+\int_{t}^{\zeta_{\tau}}\left\langle Y_{\theta}^{n}-Y_{\theta}^{*}, d K_{\theta}^{n}\right\rangle\right) .
\end{gathered}
$$

Since by (2.1) and (D3),

$$
\int_{t}^{\zeta_{\tau}}\left\langle Y_{\theta}^{n}-Y_{\theta}^{*}, d K_{\theta}^{n}\right\rangle \leq-\int_{t}^{\zeta_{\tau}} \operatorname{dist}\left(Y_{\theta}^{*}, \partial D\left(\mathbf{X}_{\theta}\right)\right) d\left|K^{n}\right|_{\theta} \leq-\varepsilon \int_{t}^{\zeta_{\tau}} d\left|K^{n}\right|_{\theta},
$$

we have

$$
\begin{aligned}
E_{S, x} & \left(\left|Y_{t}^{n}-Y_{t}^{*}\right|^{2}+\int_{t}^{\zeta_{\tau}}\left\|Z_{\theta}^{n}-\sigma \nabla u_{*}\left(\mathbf{X}_{\theta}\right)\right\|^{2} d \theta+\varepsilon \int_{t}^{\zeta_{\tau}} d\left|K^{n}\right| \theta\right) \\
\leq & E_{S, x}\left|\left(\varphi-\varphi_{*}\right)\left(\mathbf{X}_{\zeta_{\tau}}\right)\right|^{2}+C E_{S, x}\left(\int _ { t } ^ { \zeta _ { \tau } } \left\{\left|Y_{\theta}^{n}-Y_{\theta}^{*}\right|^{2}+\left|Y_{\theta}^{n}-Y_{\theta}^{*}\right| \cdot\left|Y_{\theta}^{*}\right|\right.\right. \\
& \left.\left.+\left|Y_{\theta}^{n}-Y_{\theta}^{*}\right| \cdot\left(\left\|Z_{\theta}^{n}-Z_{\theta}^{*}\right\|+\left\|Z_{\theta}^{*}\right\|\right)+\left|Y_{\theta}^{n}-Y_{\theta}^{*}\right| \cdot\left|f\left(\mathbf{X}_{\theta}, 0,0\right)-f_{*}\left(\mathbf{X}_{\theta}\right)\right|\right\} d \theta\right) .
\end{aligned}
$$

Using standard arguments one can deduce from this that

$$
\begin{aligned}
E_{s, x} & \left(\sup _{0 \leq t \leq T}\left|Y_{t}^{n}-Y_{t}^{*}\right|^{2}+\int_{0}^{\zeta_{\tau}}\left\|Z_{t}^{n}-Z_{t}^{*}\right\|^{2} d t+\varepsilon E_{s, x}\left|K^{n}\right|_{\zeta_{\tau}}\right) \\
\leq & C E_{s, x}\left(\left|\left(\varphi-\varphi_{*}\right)\left(\mathbf{X}_{\zeta_{\tau}}\right)\right|^{2}\right. \\
& \left.+\int_{0}^{\zeta_{\tau}}\left(\left|f\left(\mathbf{X}_{t}, 0,0\right)-f_{*}\left(\mathbf{X}_{t}\right)\right|^{2}+\left|Y_{t}^{*}\right|^{2}+\left\|Z_{t}^{*}\right\|^{2}\right) d t\right) .
\end{aligned}
$$

Set $\mu_{n}=\left(\mu_{n}^{1}, \ldots, \mu_{n}^{m}\right)$, where

$$
d \mu_{n}^{i}(t, y)=-n\left(u_{n}^{i}(t, y)-\left(\Pi_{D(t, y)}\left(u_{n}(t, y)\right)\right)^{i}\right) d t d y .
$$


Then

$$
d\left|\mu_{n}^{i}\right|(t, y)=n\left|u_{n}^{i}(t, y)-\left(\Pi_{D(t, y)}\left(u_{n}(t, y)\right)\right)^{i}\right| d t d y, \quad\left|\mu_{n}\right|=\sum_{i=1}^{m}\left|\mu_{n}^{i}\right| .
$$

Since $u_{*} \in L^{2}(0, T ; V)$, it follows from (3.11) and [10, Proposition 3.13] that

$$
\begin{aligned}
\varepsilon \sup _{n \geq 1}\left|\mu_{n}\right|\left(E_{0, T}\right) \leq & C\left(\left\|\varphi-\varphi_{*}\right\|_{H}^{2}+\left\|f(\cdot, \cdot, 0,0)-f_{*}\right\|_{L^{2}(0, T ; H)}^{2}\right. \\
& \left.+\left\|u_{*}\right\|_{L^{2}(0, T ; H)}^{2}+\int_{0}^{T}\left\|\nabla u_{*}(t)\right\|_{H}^{2} d t\right)
\end{aligned}
$$

Set

$$
\xi=\varphi\left(\mathbf{X}_{\zeta_{\tau}}\right), \quad \bar{f}(t, x, y)=f\left(\mathbf{X}_{t \wedge \zeta_{\tau}}, y, z\right), \quad D_{t}=D\left(\mathbf{X}_{t \wedge \zeta_{\tau}}\right), \quad t \in[0, T] .
$$

We now show that from (A1)-(A3), (D1), (D2) it follows that for q.e. ( $s, x) \in E_{0, T}$, under the measure $P_{s, x}$ the data $\xi, \bar{f}, \mathcal{D}=\left\{D_{t}, t \in[0, T]\right\}$ satisfy the following hypotheses (H1)-(H4) from [12]. In the notation of the present paper, for fixed probability measure $P_{s, x}$, these hypotheses read as follows:

(H1) $\xi \in D_{T}, E_{s, x}|\xi|^{2}<\infty$,

(H2) $E_{s, x} \int_{0}^{T}|\bar{f}(t, 0,0)|^{2} d t<\infty$,

(H3) $\bar{f}:[0, T] \times \Omega \times \mathbb{R}^{m} \times \mathbb{R}^{m \times d} \rightarrow \mathbb{R}^{m}$ is measurable with respect to Prog $\otimes$ $\mathcal{B}\left(\mathbb{R}^{m}\right) \otimes \mathcal{B}^{m \times d}$ (Prog denotes the $\sigma$-field of all progressive subsets of $[0, T] \times \Omega$ ) and there are $\alpha, \beta \geq 0$ such that $P_{s, x}$-a.s. we have

$$
\left|\bar{f}\left(t, y_{1}, z_{1}\right)-\bar{f}\left(t, y_{2}, z_{2}\right)\right| \leq \alpha\left|y_{1}-y_{2}\right|+\beta\left\|z_{1}-z_{2}\right\|
$$

for all $y_{1}, y_{2} \in \mathbb{R}^{m}$ and $z_{1}, z_{2} \in \mathbb{R}^{m \times d}$,

(H4) for each $N$ the mapping $t \mapsto D_{t} \cap\left\{x \in \mathbb{R}^{d}:|x| \leq N\right\} \in$ Conv is càdlàg $P_{s, x}$-a.s. (with the convention that $D_{T}=D_{T-}$ ), and there is a semimartingale $A \in \mathcal{H}^{2}\left(P_{s, x}\right)$ such that $A_{t} \in \operatorname{Int} D_{t}, t \in[0, T]$, and $\inf _{t \leq T} \operatorname{dist}\left(A_{t}, \partial D_{t}\right)>0$.

Clearly (A1) implies that $\xi$ satisfies (H1), and (A3) implies that $\bar{f}$ satisfies (H3) under $P_{s, x}$ for $(s, x) \in E_{T}$. To prove (H2), let us set $g(s, x)=E_{s, x} \int_{0}^{T}|\bar{f}(t, 0,0)|^{2} d t$ and $B=\left\{(s, x) \in E_{0, T}: g(s, x)=\infty\right\}$. For every $v \in \hat{S}_{00}\left(E_{0, T}\right)$ we have

$$
\int_{E_{0, T}} g d v=E_{v} \int_{0}^{\zeta_{\tau}}\left|f\left(\mathbf{X}_{t}, 0,0\right)\right|^{2} d t=\int_{E_{0, T}} R_{0}^{0, T}|f|^{2}(\cdot, \cdot, 0,0) d v .
$$

Since

$$
\int_{E_{0, T}} R_{0}^{0, T}|f|^{2}(\cdot, \cdot, 0,0) d v=\left(|f|^{2}(\cdot, \cdot, 0,0), \hat{R}_{0}^{0, T} v\right)_{L^{2}\left(0, T ; L^{2}(E)\right)}
$$


(see [10, p. 1226]), it follows from (3.13) that

$$
\int_{E_{0, T}} g d v \leq\left\|\hat{R}_{0}^{0, T} v\right\|_{\infty} \cdot\left\||f|^{2}(\cdot, \cdot, 0,0)\right\|_{L^{1}\left(0, T ; L^{1}(E)\right)}
$$

Therefore if (A2) is satisfied then $v(B)=0$ for every $v \in \hat{S}_{00}\left(E_{0, T}\right)$. By this and a known analogue of [6, Theorem 2.2.3], $\widehat{\operatorname{cap}}(B)=0$, where cap denotes the dual capacity (see [23, p. 292]). Consequently, $\operatorname{cap}(B)=0$, because $\operatorname{cap}(B)=\widehat{\operatorname{cap}}(B)$ (see [23, p. 292]). This shows that if (A2) is satisfied then (H2) holds true under $P_{s, x}$ for q.e. $(s, x) \in E_{0, T}$. That $\mathcal{D}$ satisfies (H4) under $P_{s, x}$ for q.e. $(s, x) \in E_{0, T}$ follows from (D1), (D2) and Remark 3.1. Since hypotheses (H1)-(H4) are satisfied under $P_{s, x}$ for q.e. $(s, x) \in E_{0, T}$, it follows from [12, Theorem 3.6] that for q.e. $(s, x) \in E_{0, T}$ there exists a unique solution $\left(Y^{s, x}, Z^{s, x}, K^{s, x}\right)$ of the reflected BSDE with final condition $\xi$, coefficient $\bar{f}$ and obstacle $\mathcal{D}$. This means that $Y_{t}^{s, x} \in D_{t}$ for $t \in[s, T]$,

$$
\begin{aligned}
Y_{t}^{s, x}= & \varphi\left(\mathbf{X}_{\zeta_{\tau}}\right)+\int_{t \wedge \zeta_{\tau}}^{\zeta_{\tau}} f\left(\mathbf{X}_{\theta}, Y_{\theta}^{s, x}, Z_{\theta}^{s, x}\right) d \theta \\
& +\int_{t \wedge \zeta_{\tau}}^{\zeta_{\tau}} K_{\theta}^{s, x}-\int_{t \wedge \zeta_{\tau}}^{\zeta_{\tau}} Z_{\theta}^{s, x} d B_{\theta}, \quad t \in[0, T], \quad P_{s, x} \text {-a.s. }
\end{aligned}
$$

and for every quasi-continuous $h=\left(h^{1}, \ldots, h^{m}\right)$ such that $h(t, x) \in D(t, x)$ for q.e. $(t, x) \in E_{0, T}$ we have

$$
\int_{0}^{\zeta_{\tau}}\left\langle Y_{t}^{s, x}-h\left(\mathbf{X}_{t}\right), d K_{t}^{s, x}\right\rangle \leq 0
$$

(observe that $h\left(\mathbf{X}_{\wedge \zeta_{\tau}}\right) \in D_{t}, t \in[0, T], P_{s, x}$-a.s. by Remark 3.5(i)). By [12, Theorem 4.9], for any ( $s, x)$ for which (3.10) is satisfied for all $n \in \mathbb{N}$, i.e. for q.e. $(s, x) \in E_{0, T}$,

$$
\sup _{0 \leq t \leq T}\left|Y_{t}^{n}-Y_{t}^{s, x}\right| \rightarrow 0, \quad \int_{0}^{T}\left\|Z_{t}^{n}-Z_{t}^{s, x}\right\|^{2} d t \rightarrow 0, \quad \sup _{0 \leq t \leq T}\left|K_{t}^{n}-K_{t}^{s, x}\right| \rightarrow 0
$$

in probability $P_{s, x}$ as $n \rightarrow \infty$. By (3.16) and [6, Lemma A.3.4] one can find versions $Y, K$ of $Y^{s, x}, K^{s, x}$ not depending on $s, x$ such that for q.e. $(s, x) \in E_{0, T}$,

$$
\sup _{0 \leq t \leq T}\left|Y_{t}^{n}-Y_{t}\right| \rightarrow 0, \quad \sup _{0 \leq t \leq T}\left|K_{t}^{n}-K_{t}\right| \rightarrow 0
$$

in probability $P_{s, x}$. From (3.11) and (3.17) it follows in particular that for q.e. $(s, x) \in$ $E_{0, T}$,

$$
E_{s, x}\left|u_{n}(s, x)-Y_{0}\right|=E_{s, x}\left|u_{n}\left(\mathbf{X}_{0}\right)-Y_{0}\right| \rightarrow 0,
$$


i.e. $\left\{u_{n}\right\}$ is converges q.e. in $E_{0, T}$. Let us put $u(s, x)=\lim _{n \rightarrow \infty} u_{n}(s, x)$ for those $(s, x) \in E_{0, T}$ for which the limit exists and is finite, and $u(s, x)=0$ otherwise. Then there is an $m_{1}$-version of $u$ (still denoted by $u$ ) such that $u$ is quasi-continuous and

$$
Y_{t}=u\left(\mathbf{X}_{t \wedge \zeta_{\tau}}\right), \quad t \in[0, T], \quad P_{s, x} \text {-a.s. }
$$

for q.e. $(s, x) \in E_{0, T}$. Indeed, by (3.17), $\sup _{0 \leq t \leq T}\left|u_{n}\left(\mathbf{X}_{t \wedge \zeta_{\tau}}\right)-Y_{t}\right| \rightarrow 0$ in probability $P_{s, x}$ for q.e. $(s, x) \in E_{0, T}$. On the other hand, since the set for which $\left\{u_{n}(s, x)\right\}$ does not converge is M-exceptional, it follows from Remark 2.1(ii) that $\sup _{0 \leq t \leq T} \mid u_{n}\left(\mathbf{X}_{t \wedge \zeta_{\tau}}\right)-$ $u\left(\mathbf{X}_{t \wedge \zeta_{\tau}}\right) \mid \rightarrow 0 P_{s, x}$-a.s. for q.e. $(s, x) \in E_{0, T}$. This shows (3.18). In particular, since $Y$ is continuous, [0,T] $\ni t \mapsto u\left(\mathbf{X}_{t \wedge \zeta_{\tau}}\right)$ is continuous under $P_{s, x}$ for q.e. $(s, x) \in E_{0, T}$, from which it follows that $u$ has a quasi-continuous $m_{1}$-version. Since $u_{n} \rightarrow u m_{1}$-a.e. and $u_{n}(s, \cdot) \rightarrow u(s, \cdot) m$-a.e. for every $s \in(0, T]$ [see Remark 2.1(i)], it follows from (3.5) that the first convergence in (3.8) holds true,

$$
\left\|u_{n}(s)-u(s)\right\|_{H} \rightarrow 0
$$

for every $s \in(0, T]$ and (3.7) is satisfied. Moreover, $\nabla u_{n} \rightarrow \nabla u$ weakly in $L^{2}(0, T ; H)$ as $n \rightarrow \infty$. By the Banach-Saks theorem there is a subsequence $\left(n_{k}\right)$ such that the sequence of the Cesàro mean $\bar{u}_{N}=\frac{1}{N} \sum_{k=1}^{N} u_{n_{k}}$ converges strongly, i.e.

$$
\int_{0}^{T}\left\|\nabla\left(\bar{u}_{N}-u\right)(t)\right\|_{H}^{2} d t \rightarrow 0
$$

as $N \rightarrow \infty$. For every $v \in \hat{S}_{00}\left(E_{0, T}\right)$,

$$
E_{v} \int_{0}^{T}\left\|\nabla\left(\bar{u}_{N}-u\right)\left(\mathbf{X}_{t}\right)\right\|^{2} d t \leq\left\|\hat{R}_{0}^{0, T} v\right\|_{\infty} \int_{0}^{T}\left\|\nabla\left(\bar{u}_{N}-u\right)(t)\right\|_{H}^{2} d t
$$

From (3.20), (3.21) and a known analogue of [6, Theorem 2.2.3] we conclude that $E_{S, x} \int_{0}^{T}\left\|\sigma \nabla\left(\bar{u}_{N}-u\right)\left(\mathbf{X}_{t}\right)\right\|^{2} d t \rightarrow 0$ for q.e. $(s, x) \in E_{0, T}$, On the other hand, by (3.16), $\int_{0}^{T}\left\|\frac{1}{N} \sum_{k=1}^{N} Z_{t}^{n_{k}}-Z_{t}^{s, x}\right\|^{2} d t \rightarrow 0$ in probability $P_{s, x}$ for q.e. $(s, x) \in E_{0, T}$. Consequently,

$$
\int_{0}^{T}\left\|Z_{t}^{s, x}-\sigma \nabla u\left(\mathbf{X}_{t}\right)\right\|^{2} d t=0, \quad P_{s, x} \text {-a.s. }
$$

for q.e. $(s, x) \in E_{0, T}$. From this and (3.16) we deduce that (3.20) holds with $\bar{u}_{N}$ replaced by $u_{n}$, i.e. the second part of (3.8) is satisfied. By (3.12) there is a subsequence (still denoted by $n$ ), and a bounded measure $\mu$ on $(0, T] \times E$ such that (3.9) is satisfied. By (2.7), for any $f \in C_{c}((s, T) \times E$ and $i=1, \ldots, m$ we have

$$
E_{s, x} \int_{0}^{\zeta_{\tau}} f\left(\mathbf{X}_{t}\right) d K_{t}^{n, i}=\int_{s}^{T} \int_{E} f(t, y) p_{E}(s, x, t, y) d \mu_{n}^{i}(t, y)
$$


for q.e. $(s, x) \in E_{0, T}$. Letting $n \rightarrow \infty$ and using (3.17), (3.9) and the fact that $(s, T) \times E \ni(t, y) \rightarrow p_{E}(s, x, t, y)$ is continuous we obtain

$$
E_{s, x} \int_{0}^{\zeta_{\tau}} f\left(\mathbf{X}_{t}\right) d K_{t}^{i}=\int_{s}^{T} \int_{E} f(t, y) p_{E}(s, x, t, y) d \mu^{i}(t, y), \quad i=1, \ldots, m .
$$

Hence $K=A^{\mu}$ [see (2.7)]. Thus $\left(Y, Z, A^{\mu}\right)$ is a version of $\left(Y^{s, x}, Z^{s, x}, K^{s, x}\right)$ for q.e. $(s, x) \in E_{0, T}$. In particular, by (3.14) and (3.15),

$$
\begin{aligned}
Y_{t}= & \varphi\left(\mathbf{X}_{\zeta_{\tau}}\right)+\int_{t \wedge \zeta_{\tau}}^{\zeta_{\tau}} f\left(\mathbf{X}_{\theta}, Y_{\theta}, Z_{\theta}\right) d \theta \\
& +\int_{t \wedge \zeta_{\tau}}^{\zeta_{\tau}} A_{\theta}^{\mu}-\int_{t \wedge \zeta_{\tau}}^{\zeta_{\tau}} Z_{\theta} d B_{\theta}, \quad t \in[0, T], \quad P_{s, x} \text {-a.s. }
\end{aligned}
$$

and

$$
\int_{0}^{\zeta_{\tau}}\left\langle Y_{t}-h\left(\mathbf{X}_{t}\right), d A_{t}^{\mu}\right\rangle \leq 0
$$

for q.e. $(s, x) \in E_{0, T}$. When combined with (3.18) and (3.22) this proves that $(u, \mu)$ satisfies (3.1), (3.2). What is left is to show that $\mu \in S\left(E_{0, T}\right)$. To prove this, for fixed $s \in(0, T)$ set $\varrho(s ; t, y)=\int_{E} p_{E}(s, y, t, x) d x$. Observe that $(s, T] \times E \ni$ $(t, y) \mapsto v(s, y):=\varrho(s ; t, y)$ is a weak solution of the Cauchy-Dirichlet problem $\left(\frac{\partial}{\partial t}-L_{t}\right) v=0$ in $(s, T] \times E,\left.v\right|_{(s, T] \times \partial E}=0, v(s)=1$ (see [1, Sect. 8]). In particular, $v$ is strictly positive and continuous on $(s, T] \times E$. Let $B$ be a Borel subset of $(s, T] \times E$ such that $\operatorname{cap}(B)=0$, and let $H$ be a compact subset of $B$. By an approximation argument, (3.23) holds true for every bounded Borel function $f$ on $(s, T] \times E$. In particular, taking $f=\mathbf{1}_{H}$ in (3.23) we get

$$
\begin{aligned}
\int_{H} \varrho(s ; t, y) d \mu^{i}(t, y) & =\int_{E}\left(\int_{H} p_{E}(s, x, t, y) d \mu^{i}(t, y)\right) d x \\
& =\int_{E}\left(E_{s, x} \int_{0}^{\zeta_{\tau}} \mathbf{1}_{H}\left(\mathbf{X}_{t}\right) d K_{t}^{i}\right) d x=0,
\end{aligned}
$$

the last equality being a consequence of (2.6). Since inf $(t, y) \in H \varrho(s ; t, y)>0$, it follows that $\mu^{i}(H)=0$. Since $s \in(0, T)$ was arbitrary, $\mu^{i}(H)=0$ for every compact subset $H$ of $E_{0, T}$. This and [23, (4.4)]) imply that $\mu(B)=0$. Thus $\mu$ charges no set of capacity zero. Hence $\mu \in \mathcal{M}_{0, b}\left(E_{0, T}\right)$, which completes the proof.

It is perhaps worth remarking that in case the operator $L$ is in nondivergence form [for instance, $L$ is the Laplace operator $\Delta$, or, more generally, $\frac{\partial a_{i j}}{\partial x_{k}} \in L^{\infty}((0, T) \times E)$ for $i, j, k=1, \ldots, d]$, then the process $\mathbf{X}$ corresponding to $L$ can be constructed by solving an Itô equation. This allows simplifying some arguments in the proofs of the results presented above, but actually not much. One reason is that we are working 
in $L^{2}$ setting, so even in the case where $L=\Delta$ the solution of (1.1), (1.2) need not be continuous. On the other hand, since we use a stochastic approach via BSDEs [the basic relation is $Y=u(\mathbf{X})$, where $Y$ is the first component of the solution of the corresponding BSDE; see Remark 3.5(ii)], we must know that $Y$ is continuous, and hence that $u$ is quasi-continuous. This in turn requires the introduction of quasinotions (capacity, quasi-continuity, etc.) and we still have to use some results from the probabilistic potential theory.

\section{Variational Solutions}

In this section we show that results of Sect. 3 can be translated into results on solutions of (1.1), (1.2) in the sense of the analytical definition formulated below. Solutions in the sense of this definition will be called variational solutions.

Definition 4.1 We say that a pair $(u, \mu)$, where $u=\left(u^{1}, \ldots, u^{m}\right): E_{T} \rightarrow \mathbb{R}^{m}$ and $\mu=\left(\mu^{1}, \ldots \mu^{m}\right)$ is a signed Borel measure on $E_{0, T}$ is a variational solution of $\mathrm{OP}(\varphi, f, D)$ if

(a) $u$ is quasi-continuous, $u^{i} \in C([0, T] ; H) \cap L^{2}\left(0, T ; H_{0}^{1}(E)\right), \mu^{i} \in \mathcal{M}_{0, b}\left(E_{0, T}\right)$, $i=1, \ldots, m$,

(b) for every $\eta=\left(\eta^{1}, \ldots, \eta^{m}\right)$ such that $\eta^{i}$ is bounded and $\eta^{i} \in \mathcal{W}_{0}$ for $i=1, \ldots, m$ we have

$$
\begin{gathered}
(u(t), \eta(t))_{H}+\int_{t}^{T}\left\langle\frac{\partial \eta}{\partial s}(s), u(s)\right\rangle_{V^{\prime}, V} d s+\int_{t}^{T} a^{(s)}(u(s), \eta(s)) d s \\
=(\varphi, \eta(T))_{H}+\int_{t}^{T}\left(f_{u}(s), \eta(s)\right)_{H} d s+\int_{t}^{T} \int_{E}\langle\eta, d \mu\rangle
\end{gathered}
$$

for all $t \in[0, T]$, where $\left\{a^{(t)}(\cdot, \cdot), t \geq 0\right\}$ is the family of bilinear forms on $V \times V$ defined as

$$
a^{(t)}(\varphi, \psi)=\frac{1}{2} \sum_{k=1}^{m} \sum_{i, j=1}^{d} \int_{E} a_{i j}(t, x) \frac{\partial \varphi^{k}}{\partial x_{i}} \frac{\partial \psi^{k}}{\partial x_{j}} d x
$$

(c) $u(t, x) \in D(t, x)$ for q.e. $(t, x) \in E_{0, T}$, and for every quasi-continuous function $h=\left(h^{1}, \ldots, h^{m}\right)$ such that $h(t, x) \in D(t, x)$ for q.e. $(t, x) \in E_{0, T}$,

$$
\int_{t}^{T} \int_{E}\langle u-h, d \mu\rangle \leq 0
$$

for all $t \in(0, T)$. 
Remark 4.2 If condition (b) of Definition 4.1 is satisfied then for any bounded $v \in \mathcal{W}_{0}$ we have

$$
\mathcal{E}^{0, T}\left(u^{i}, \eta\right)=\left(\varphi^{i}, \eta(T)\right)_{H}+\int_{0}^{T}\left(f_{u}^{i}(s), \eta(s)\right)_{H} d s+\int_{E_{0, T}} \eta d \mu^{i}, \quad i=1, \ldots, m .
$$

To see this it suffices to take $t=0$ in (4.1) and consider $\eta$ such that $\eta^{i}=v, \eta^{j}=0$ for $j \neq i$. Also note that using a standard argument (see, e.g., the reasoning following (1.16) in [16, Chap. III]) and the fact that $\mu(\{t\} \times E)=0$ for $t \in[0, T]$ [see Remark 3.5(iv)] one can show that (4.3) implies (4.1).

Remark 4.3 Assume that $m=1$ and $D(t, x)=\{y \in \mathbb{R}: \underline{h}(t, x) \leq y \leq \bar{h}(t, x)\}$ for some quasi-continuous $\underline{h}, \bar{h}: E_{T} \rightarrow \mathbb{R}$ such that $\underline{h}<\bar{h}$. Then (4.2) reduces to the condition $\int_{E_{0, T}}(u-\underline{h}) \bar{d} \mu^{+}=\int_{E_{0, T}}(\bar{h}-u) d \mu^{-}=0$, where $\mu^{+}\left(\right.$resp. $\left.\mu^{-}\right)$is the positive (resp. negative) part of the Jordan decomposition of $\mu$. The case of merely measurable obstacles is discussed in $[8,9,11]$.

Proposition 4.4 Assume (A3), (D1). Then there exists at most one variational solution of $\mathrm{OP}(\varphi, f, D)$.

Proof Suppose that $\left(u_{1}, \mu_{1}\right),\left(u_{2}, \mu_{2}\right)$ are solutions of $\operatorname{OP}(\varphi, f, D)$ and set $u=u_{1}-$ $u_{2}, \mu=\mu_{1}-\mu_{2}$. Then from (4.1) it follows that for every bounded quasi-continuous $\eta \in \mathcal{W}_{0}$,

$$
\begin{array}{r}
(u(t), \eta(t))_{H}+\int_{t}^{T}\left\langle\frac{\partial \eta}{\partial s}, u(s)\right\rangle_{V^{\prime}, V} d s+\int_{t}^{T} a^{(s)}(u(s), \eta(s)) d s \\
=\int_{t}^{T}\left(f_{u_{1}}(s)-f_{u_{2}}(s), \eta(s)\right)_{H} d s+\int_{t}^{T} \int_{E}\left\langle\eta, d\left(\mu_{1}-\mu_{2}\right)\right\rangle .
\end{array}
$$

Set $u_{\alpha}^{i}=\alpha \hat{R}_{\alpha}^{0, T} u^{i}, i=1, \ldots, m$, where $\hat{R}_{\alpha}^{0, T} u^{i}$ is defined by (3.6) with $\mu=u^{i} d m_{1}$. By [10, (3.17)], $\hat{R}_{\alpha}^{0, T} u^{i}$ is an $m_{1}$-version of $\hat{G}_{\alpha}^{0, T} u^{i}$, where $\left(\hat{G}_{\alpha}^{0, T}\right)_{\alpha>0}$ is the coresolvent associated with the form $\mathcal{E}^{0, T}$ (see (2.5)). Taking $\eta=u_{\alpha}=\left(u_{\alpha}^{1}, \ldots, u_{\alpha}^{m}\right)$ as test function in (4.4) we obtain

$$
\begin{gathered}
\left(u(t), u_{\alpha}(t)\right)_{H}+\int_{t}^{T}\left\langle\frac{\partial u_{\alpha}}{\partial s}(s), u(s)\right\rangle_{V^{\prime}, V} d s+\int_{t}^{T} a^{(s)}\left(u(s), u_{\alpha}(s)\right) d s \\
=\int_{t}^{T}\left(f_{u_{1}}(s)-f_{u_{2}}(s), u_{\alpha}(s)\right)_{H} d s+\int_{t}^{T} \int_{E}\left\langle u_{\alpha}, d\left(\mu_{1}-\mu_{2}\right)\right\rangle .
\end{gathered}
$$

The generator $\hat{\mathcal{L}}$ of the co-resolvent $\left(\hat{G}_{\alpha}^{0, T}\right)_{\alpha>0}$ has the form $(\hat{\mathcal{L}} v)(s)=-\frac{\partial v}{\partial s}(s)+$ $L_{t} v(s)$ for $v \in \mathcal{W}_{0}$. Hence $\left(\alpha-\left(-\frac{\partial}{\partial s}+L_{s}\right)\right) \hat{R}_{\alpha}^{0, T} u=u$, and consequently

$$
\left(-\frac{\partial}{\partial s}+L_{s}\right) u_{\alpha}=\alpha\left(u_{\alpha}-u\right)
$$


Therefore

$\int_{t}^{T}\left\langle\frac{\partial u_{\alpha}}{\partial s}(s), u(s)\right\rangle_{V^{\prime}, V} d s+\int_{t}^{T} a^{(s)}\left(u(s), u_{\alpha}(s)\right) d s=\int_{t}^{T} \alpha\left(u(s)-u_{\alpha}(s), u(s)\right) d s$

and

$$
\begin{gathered}
\frac{1}{2}\left\|u_{\alpha}(T)\right\|_{H}^{2}-\frac{1}{2}\left\|u_{\alpha}(t)\right\|_{H}^{2}+\int_{t}^{T} a^{(s)}\left(u_{\alpha}(s), u_{\alpha}(s)(s)\right) d s \\
=\int_{t}^{T} \alpha\left(u(s)-u_{\alpha}(s), u_{\alpha}(s)\right) d s .
\end{gathered}
$$

By the last two equalities,

$$
\begin{aligned}
& \int_{t}^{T}\left\langle\frac{\partial u_{\alpha}}{\partial s}(s), u(s)\right\rangle_{V^{\prime}, V} d s+\int_{t}^{T} a^{(s)}\left(u(s), u_{\alpha}(s)\right) d s \\
& \quad=\int_{t}^{T} \alpha\left(u(s)-u_{\alpha}(s), u(s)-u_{\alpha}(s)\right) d s+\int_{t}^{T} \alpha\left(u(s)-u_{\alpha}(s), u_{\alpha}(s)\right) d s \\
& \quad \geq \int_{t}^{T} a^{(s)}\left(u_{\alpha}(s), u_{\alpha}(s)\right) d s+\frac{1}{2}\left\|u_{\alpha}(T)\right\|_{H}^{2}-\frac{1}{2}\left\|u_{\alpha}(t)\right\|_{H}^{2} .
\end{aligned}
$$

Since $u \in L^{2}(0, T ; V)$ and it is known that $\left(\hat{G}_{\alpha}^{0, T}\right)$ is strongly continuous (see, e.g., [28, Proposition I.3.7]), $u_{\alpha} \rightarrow u$ in $L^{2}(0, T ; V)$ as $\alpha \rightarrow \infty$. Hence, for every $t \in[0, T]$,

$$
\int_{t}^{T} a^{(s)}\left(u_{\alpha}(s), u_{\alpha}(s)\right) d s \rightarrow \int_{t}^{T} a^{(s)}(u(s), u(s)) d s .
$$

Since $u$ is quasi-continuous, [0,T] $\ni t \mapsto u\left(\hat{\mathbf{X}}_{t}\right)$ is continuous $P_{s, x}$-a.s. for q.e. $(s, x) \in E_{0, T}$. Hence $\alpha \int_{0}^{\hat{\zeta} \wedge \tau(0)} e^{-\alpha t} u\left(\hat{\mathbf{X}}_{t}\right) d t=\int_{0}^{\alpha(\hat{\zeta} \wedge \tau(0))} e^{-t} u\left(\hat{\mathbf{X}}_{t / \alpha}\right) d t \rightarrow u\left(\mathbf{X}_{0}\right)$ $P_{s, x}$-a.s. as $\alpha \rightarrow \infty$ for q.e. $(t, x) \in E_{0, T}$. Since $u(t, y) \in D(t, y)$ for q.e. $(t, y) \in E_{0, T}$ and the sets $D(t, y)$ are uniformly bounded, it follows from this and the Lebesgue dominated convergence theorem that $u_{\alpha}(s, x) \rightarrow E_{s, x} u\left(\mathbf{X}_{0}\right)=u(s, x)$ for q.e. $(s, x) \in E_{0, T}$. Hence, by the Lebesgue dominated convergence theorem again,

$$
\left(u(t), u_{\alpha}(t)\right)_{H} \rightarrow\|u(t)\|_{H}^{2}, \quad\left\|u_{\alpha}(t)\right\|_{H}^{2} \rightarrow\|u(t)\|_{H}^{2}
$$

and

$$
\int_{t}^{T} \int_{E}\left\langle u_{\alpha}, d\left(\mu_{1}-\mu_{2}\right)\right\rangle \rightarrow \int_{t}^{T} \int_{E}\left\langle u, d\left(\mu_{1}-\mu_{2}\right)\right\rangle
$$

By (4.2), the right-hand side of (4.9) is nonpositive. Therefore letting $\alpha \rightarrow \infty$ in (4.5) and using (4.6)-(4.9) we get 
$\frac{1}{2}\|u(t)\|_{H}^{2}+\int_{t}^{T} a^{(s)}(u(s), u(s)) d s \leq \int_{t}^{T}\left(f_{u_{1}}(s)-f_{u_{2}}(s), u(s)\right)_{H} d s, \quad t \in[0, T]$.

Using (A3) and Gronwall's lemma we deduce from the above inequality that $u=0$ a.e.

Theorem 4.5 Under the assumptions of Theorem 3.8 there exists a unique variational solution of $\mathrm{OP}(\varphi, f, D)$.

Proof We need only prove the existence of a solution. By Theorem 3.8 there exists a probabilistic solution $(u, \mu)$ of $\mathrm{OP}(\varphi, f, D)$. By (3.7) and Remark 3.5(iii), $u \in$ $C([0, T] ; H)$, and hence $(u, \mu)$ satisfies condition (a) of Definition 4.1. To see that (b) and (c) are satisfied, let us consider solutions $u_{n}$ of (3.4). Then for every $\eta=$ $\left(\eta^{1}, \ldots, \eta^{m}\right)$ such that $\eta_{i} \in C_{c}\left(E_{0, T}\right)$ for $i=1, \ldots, m$ we have

$$
\begin{gathered}
\left(u_{n}(t), \eta(t)\right)_{H}+\int_{t}^{T}\left\langle\frac{\partial \eta}{\partial s}(s), u_{n}(s)\right\rangle_{V^{\prime}, V} d s+\int_{t}^{T} a^{(s)}\left(u_{n}(s), \eta(s)\right) d s \\
=(\varphi, \eta(T))_{H}+\int_{t}^{T}\left(f_{u_{n}}(s), \eta(s)\right)_{H} d s+\int_{t}^{T} \int_{E} \eta d \mu_{n},
\end{gathered}
$$

Letting $n \rightarrow \infty$ in the above equality and using (3.8), (3.9), (3.19) and (A3) we conclude that (4.1) is satisfied for $\eta$ as above, and hence for $\eta \in \mathcal{W}_{0}$ since $C_{c}\left(E_{0, T}\right)$ is dense is $\mathcal{W}_{0}$. Moreover, by (2.7) and (3.2), for every quasi-continuous $h$ such that $h(t, x) \in D(t, x)$ for q.e. $(t, x) \in E_{0, T}$ we have

$$
\begin{aligned}
& \sum_{i=1}^{m} \int_{s}^{T} \int_{E}\left(u^{i}-h^{i}\right)(t, y) p_{E}(s, x, t, y) d \mu^{i}(t, y) \\
& \quad=E_{s, x} \int_{0}^{\zeta_{\tau}}\left\langle u\left(\mathbf{X}_{t}\right)-h\left(\mathbf{X}_{t}\right), d A_{t}^{\mu}\right\rangle \leq 0
\end{aligned}
$$

for q.e. ( $s, x) \in E_{0, T}$. Integrating with respect to $x$ and using Remark 2.1(i) we obtain

$$
\sum_{i=1}^{m} \int_{s}^{T} \int_{E}\left(u^{i}-h^{i}\right)(t, y) \varrho(s ; t, y) d \mu^{i}(t, y) \leq 0
$$

for every $s \in(0, T]$, which implies $(4.2)$, because $\varrho(s ; \cdot, \cdot)$ is strictly positive.

Remark 4.6 Let the assumptions of Theorem 3.8 hold. Then the first component $u$ of the solution of $\mathrm{OP}(\varphi, f, D)$ satisfies the following parabolic variational inequality: for every $v \in \mathcal{W}$ such that $v(t, x) \in D(t, x)$ for a.e. $(t, x) \in E_{T}$,

$$
\begin{gathered}
\int_{0}^{T}\left\langle\frac{\partial v}{\partial t}(t), v(t)-u(t)\right\rangle_{V^{\prime}, V} d t-\int_{0}^{T} a^{(t)}(u(t), v(t)-u(t)) d t \\
+\int_{0}^{T}\left(f_{u}(t), v(t)-u(t)\right)_{H} d t \leq \frac{1}{2}\|v(T)-\varphi\|_{H}^{2} .
\end{gathered}
$$


To see this, let us consider the solution $u_{n}$ of (3.4). Taking $v-u_{n}$ as test function in (3.4) we get

$$
\begin{gathered}
\int_{0}^{T}\left\langle\frac{\partial u_{n}}{\partial t}, v(t)-u_{n}(t)\right\rangle_{V^{\prime}, V} d t-\int_{t}^{T} a^{(t)}\left(u_{n}(t), v(t)-u_{n}(t)\right) d t \\
=-\int_{0}^{T}\left(f_{u_{n}}(t), v(t)-u_{n}(t)\right)_{H} d t-\int_{E_{0, T}}\left\langle v-u_{n}, d \mu_{n}\right\rangle
\end{gathered}
$$

Since

$$
\begin{aligned}
\int_{0}^{T}\left\langle\frac{\partial u_{n}}{\partial t}(t), v(t)-u_{n}(t)\right\rangle_{V^{\prime}, V} d t= & \int_{0}^{T}\left\langle\frac{\partial v}{\partial t}(t), v(t)-u_{n}(t)\right\rangle_{V^{\prime}, V} d t \\
& -\frac{1}{2}\|v(T)-\varphi\|_{H}^{2}+\frac{1}{2}\left\|v(0)-u_{n}(0)\right\|_{H}^{2}
\end{aligned}
$$

it follows from (4.11) that

$$
\begin{aligned}
\int_{0}^{T}\langle & \left.\frac{\partial v}{\partial t}(t), v(t)-u_{n}(t)\right\rangle_{V^{\prime}, V} d t-\int_{0}^{T} a^{(t)}\left(u_{n}(t), v(t)-u_{n}(t)\right) d t \\
& +\int_{0}^{T}\left(f_{u_{n}}(t), v(t)-u_{n}(t)\right)_{H} d t \\
\quad= & \frac{1}{2}\|v(T)-\varphi\|_{H}^{2}-\frac{1}{2}\|v(0)-u(0)\|_{H}^{2}-\int_{E_{0, T}}\left\langle v-u_{n}, d \mu_{n}\right\rangle \leq \frac{1}{2}\|v(T)-\varphi\|_{H}^{2},
\end{aligned}
$$

the last inequality being a consequence of (2.2). Letting $n \rightarrow \infty$ and using (3.8), (3.9) and (A3) we get (4.10).

We close this section with stating some open problems. In the whole paper we assume that $E$ is bounded. Therefore our results do not apply to single equations with one obstacle or, for instance, to systems of equations with obstacles of the form

$$
D(t, x)=\left\{y \in \mathbb{R}^{m}: y_{i} \geq h^{i}(t, x)\right\} \quad \text { or } \quad D(t, x)=\left\{y \in \mathbb{R}^{m}: y_{i} \leq h^{i}(t, x)\right\} .
$$

It would be desirable to extend our results to the setting which cover these examples. By the way, note that in the paper [12], on which we rely, $E$ need not be bounded [see condition (H4) formulated in the proof of Theorem 3.8]. Another natural problem is to generalize the results of the present paper to systems involving more general operators, for instance nonsymmetric operators of the form $L_{t}^{b}=L_{t}+\sum_{i=1}^{d} b_{i}(x) \frac{\partial}{\partial x_{i}}$ with bounded measurable $b:[0, T] \times E \rightarrow \mathbb{R}^{d}$. It is worth noting here that in [20, Sect. 1.2] the existence of solutions of variational inequalities with obstacles of the form (4.12) and operator $L_{t}^{b}$ is proved in the special case where $f=\left(f^{1}, \ldots, f^{m}\right)$ and $f^{i}(t, x, y)=\sum_{j=1}^{m} c_{i j}(t, x) y_{j}$ with bounded measurable $c_{i j}$ such that $c_{i j} \geq 0$ a.e. for $i \neq j$ (in fact, in [20], the principal part and the first order part of the operator 
need not be the same in each equation of the system). Still another problem of interest is to generalize the results of the paper to irregular obstacles and/or $L^{1}$ data (for one dimensional results in this direction see $[8,9,11])$.

\section{Acknowledgements This research was supported by NCN Grant No. 2012/07/B/ST1/03508.}

Open Access This article is distributed under the terms of the Creative Commons Attribution 4.0 International License (http://creativecommons.org/licenses/by/4.0/), which permits unrestricted use, distribution, and reproduction in any medium, provided you give appropriate credit to the original author(s) and the source, provide a link to the Creative Commons license, and indicate if changes were made.

\section{References}

1. Aronson, D.G.: Non-negative solutions of linear parabolic equations. Ann. Scuola Norm. Super. Pisa 22, 607-693 (1968)

2. Bensoussan, A., Lions, J.-L.: Applications of Variational Inequalities in Stochastic Control. NorthHolland, Amsterdam (1982)

3. Blumenthal, R.M., Getoor, R.K.: Markov Processes and Potential Theory. Academic Press, New York (1968)

4. Chen, Q.: Optimal obstacle control problem for semilinear evolutionary bilateral variational inequalities. J. Math. Anal. Appl. 307, 677-690 (2005)

5. Duvaut, G., Lions, J.-L.: Inequalities in Mechannics and Physics. Springer, Berlin (1976)

6. Fukushima, M., Oshima, Y., Takeda, M.: Dirichlet Forms and Symmetric Markov Processes. Second revised and extended edition. Walter de Gruyter, Berlin (2011)

7. Gegout-Petit, A., Pardoux, É.: Equations différentielles stochastiques rétrogrades réfléchies dans un convexe. Stoch. Stoch. Rep. 57, 111-128 (1996)

8. Klimsiak, T.: Reflected BSDEs and the obstacle problem for semilinear PDEs in divergence form. Stoch. Process. Appl. 122, 134-169 (2012)

9. Klimsiak, T.: Cauchy problem for semilinear parabolic equation with time-dependent obstacles: a BSDEs approach. Potential Anal. 39, 99-140 (2013)

10. Klimsiak, T.: Semi-Dirichlet forms, Feynman-Kac functionals and the Cauchy problem for semilinear parabolic equations. J. Funct. Anal. 268, 1205-1240 (2015)

11. Klimsiak, T., Rozkosz, A.: Obstacle problem for semilinear parabolic equations with measure data. J. Evol. Equ. 15, 457-491 (2015)

12. Klimsiak, T., Rozkosz, A., Słomiński, L.: Reflected BSDEs in time-dependent convex regions. Stoch. Process. Appl. 125, 571-596 (2015)

13. Kubo, M.: Variational inequalities with time-dependent constraints in $L^{p}$. Nonlinear Anal. 73, 390-398 (2010)

14. Kubo, M., Shirakawa, K., Yamazaki, N.: Variational inequalities for a system of elliptic-parabolic equations. J. Math. Anal. Appl. 387, 490-511 (2012)

15. Kubo, M., Yamazaki, N.: Periodic solutions of elliptic-parabolic variational inequalities with timedependent constraints. J. Evol. Equ. 6, 71-93 (2006)

16. Ladyženskaja, O.A., Solonnikov, V.A., Ural'ceva, N.N.: Linear and Quasi-linear Equations of Parabolic Type. Translations of Mathematical Monographs, vol. 23. American Mathematical Society, Providence, RI (1968)

17. Lions, J.-L.: Contrôle optimal de systèmes gouvernés par des équations aux dérivées partielles. Dunod, Paris (1968)

18. Lions, J.-L.: Quelques méthodes de résolution des problèmes aux limites non linéares. Dunod, Paris (1969)

19. Menaldi, J.L.: Stochastic variational inequality for reflected diffusion. Indiana Univ. Math. J. 32, 733744 (1983)

20. Mignot, F., Puel, J.P.: Inéquations d'Evolution Paraboliques avec Convexes dépendant du Temps. Applications aux Inéquations Quasi-Variationnelles d'Evolution. Arch. Ration. Mech. Anal. 64, 59$91(1997)$ 
21. Monteiro Marques, M.: Differential Inclusions in Nonsmooth Mechanical Problems. Shocks and Dry Friction. Birkhäuser, Basel (1993)

22. Nyström, K., Olofsson, M.: Reflected BSDE of Wiener-Poisson type in time-dependent domains. Stoch. Models 32, 275-300 (2016)

23. Oshima, Y.: Time-dependent Dirichlet forms and related stochastic calculus. Infin. Dimens. Anal. Quant. Probab. Relat. Top. 7, 281-316 (2004)

24. Oshima, Y.: Semi-Dirichlet Forms and Markov Processes. Walter de Gruyter, Berlin (2013)

25. Ouknine, Y.: Reflected backward stochastic differential equations with jumps. Stoch. Stoch. Rep. 65, 111-125 (1998)

26. Pierre, M.: Problemes d'Evolution avec Contraintes Unilatérales et Potentiel Paraboliques. Commun. Partial Differ. Equ. 4, 1149-1197 (1979)

27. Protter, P.: Stochastic Integration and Differential Equations, 2nd edn. Springer, Berlin (2004)

28. Stannat, S.: The theory of generalized Dirichlet forms and its applications in analysis and stochastics. Mem. Am. Math. Soc., vol. 142(678) (1999)

29. Trutnau, G.: Stochastic calculus of generalized Dirichlet forms and applications to stochastic differential equations in infinite dimensions. Osaka J. Math. 37, 315-343 (2000) 